MATHEMATICS OF COMPUTATION

Volume 74, Number 251, Pages 1117-1138

S 0025-5718(04)01697-7

Article electronically published on August 10, 2004

\title{
A POSTERIORI ANALYSIS OF THE FINITE ELEMENT DISCRETIZATION OF SOME PARABOLIC EQUATIONS
}

\author{
A. BERGAM, C. BERNARDI, AND Z. MGHAZLI
}

\begin{abstract}
We are interested in the discretization of parabolic equations, either linear or semilinear, by an implicit Euler scheme with respect to the time variable and finite elements with respect to the space variables. The main result of this paper consists of building error indicators with respect to both time and space approximations and proving their equivalence with the error, in order to work with adaptive time steps and finite element meshes.

RÉSumÉ. Nous considérons la discrétisation d'équations paraboliques, soit linéaires soit semi-linéaires, par un schéma d'Euler implicite en temps et par éléments finis en espace. L'idée de cet article est de construire des indicateurs d'erreur liés à l'approximation en temps et en espace et de prouver leur équivalence avec l'erreur, dans le but de travailler avec des pas de temps adaptatifs et des maillages d'éléments finis adaptés à la solution.
\end{abstract}

\section{INTRODUCTION}

For more than twenty years, an impressive amount of work has been done concerning a posteriori analysis and mesh adaptivity for the finite element discretization of elliptic problems; see [Ve1] and references therein. The main results consist of exhibiting local error indicators which can be computed explicitly as a function of the discrete solution and the data and such that their Hilbertian sum is equivalent to the error. Moreover, since they are local, they provide a good representation of the error distribution; hence they are very efficient tools for mesh adaptivity. However, it seems that the analogous results concerning parabolic problems are presently not complete. They most often deal either only with time scheme adaptivity (see [JNT] or [NSV]) or space finite element adaptivity (see for instance [BB1, [BB2, [BBHM] or [BM]) or with space-time finite element adaptivity (see [E.J1], E.J2], Ve2], Ve3] and [Ve5]): the finite element discretization in these references relies on the space-time variational formulation of the equation and this leads to a family of error indicators which represent the combined space and time errors.

In this paper, we are interested in the discretization of some parabolic equations, which relies on a standard finite element method with respect to the space variables and Euler's implicit scheme with respect to time. Even if the same discrete problem is already considered in [Pi] and $[\mathrm{Ve} 5]$, our adaptivity strategy is rather different.

Received by the editor January 19, 2002 and, in revised form, January 27, 2004.

2000 Mathematics Subject Classification. Primary 65N30, 65N50.

Key words and phrases. Parabolic equations, finite elements, a posteriori analysis.

Recherche menée dans le cadre du projet AUPELF-UREF n ${ }^{0}$ 2000/PAS/38 et de l'A.I. FranceMaroc $n^{0} 221 / \mathrm{STU} / 00$

(C) 2004 American Mathematical Society 
Indeed, we follow a new approach where the main idea consists of uncoupling as much as possible the time and space errors. To this aim, we introduce two kinds of indicators, both of them of residual type. The first family, which is similar to that introduced in [JNT] or [Pi], is global with respect to the space variables but local with respect to the time discretization: thus, at each time, the error indicator provides appropriate information for the choice of the next time step. The second family is local with respect to both the time and space variables, and the idea is that at each time it is an efficient tool for mesh adaptivity. Indeed, the aim is to perform time and space adaptivity in two successive steps:

- Once the solution at a fixed time is known, we compute the time error indicator. If it is larger than a given tolerance, we go back to the previous time and make a new computation with a smaller time step till this indicator becomes smaller than the tolerance.

- Next we perform mesh adaptivity in a standard way, by computing the space error indicators, refining the mesh where they are larger than their mean value and possibly coarsening the mesh where they are much smaller.

It turns out that computing the two kinds of indicators is not very expensive in most practical situations, for instance when low degree polynomials are used in the finite element approximation. However this strategy leads us to use different triangulations at the different time steps, which of course makes the analysis of the error more complex.

Once these error indicators are constructed, we prove optimal error bounds, in the sense of [BMV]: up to some terms only involving the data, the error is bounded from above and from below by the Hilbertian sum of the indicators multiplied by constants independent of the discretization parameters. Moreover, as usual, each indicator is bounded by the local error. We first prove these results for a linear heat equation with a diffusion coefficient depending on the space and time variables (this equation is already considered in [BBHM], where mesh adaptivity is performed via a nonconforming mortar finite element technique).

Next, we extend the results to a semilinear heat equation where the diffusion coefficient now depends on the solution, as already considered in [EJ2], [NSV] and Ve2], Ve3], Ve5]. Some arguments for the extension of a posteriori analysis to nonlinear problems have been proposed in $[\overline{\mathrm{PR}}]$ and $[\mathrm{Ve} 5]$; however they do not seem appropriate for the present problem and for the kinds of indicators we work with. For the same reason, the a priori analysis in $[\overline{B R}]$ does not fit this problem and we refer to [Th] for the numerical analysis of the finite element discretization in a simple case. The equivalence of the Hilbertian sum of both types of indicators with the error is derived simply by extending the arguments of the linear case, and we skip the proofs since they do not involve any new argument.

An outline of the paper is as follows.

- In Section 2, we present the linear heat equation. We describe first its time semi-discretization and next its space and time discretization.

- Section 3 is devoted to the construction of error indicators for this equation and to the proof of upper and lower bounds for the error as a function of these indicators.

- In Section 4, these results are extended to the semilinear heat equation. 


\section{The Linear heAT EQUATION AND its Discretization}

We first present the linear heat equation with a diffusion coefficient depending on the space and time variables. Next we describe the time semi-discrete problem and finally the full discrete problem. At each step, we prove stability estimates and we recall some a priori error estimates for both the semi-discrete and discrete problems.

2.1. The linear heat equation. Let $\Omega$ be a connected and bounded open set in $\mathbb{R}^{d}, d=2$ or 3 , with a Lipschitz-continuous boundary. In order to avoid curved elements, we assume that $\Omega$ is a polygon or a polyhedron. Also let $T$ be a fixed positive integer. We consider the heat equation

$$
\begin{cases}\partial_{t} u-\operatorname{div}(\lambda \nabla u)=f & \text { in } \Omega \times] 0, T[, \\ u=0 & \text { on } \partial \Omega \times] 0, T[, \\ u_{\mid t=0}=u_{0} & \text { in } \Omega,\end{cases}
$$

where $\lambda$ is a given continuous function on $\bar{\Omega} \times[0, T]$ satisfying for some positive constants $\lambda_{\min }$ and $\lambda_{\max }$,

$$
\forall \boldsymbol{x} \in \bar{\Omega}, \forall t \in[0, T], \quad \lambda_{\min } \leq \lambda(\boldsymbol{x}, t) \leq \lambda_{\max }
$$

The data are the distribution $f$ and the function $u_{0}$; the unknown is the function $u$.

We use the standard spaces $L^{p}(\Omega), 1 \leq p \leq \infty$, together with the Sobolev spaces $H^{\mathrm{S}}(\Omega)$, where $\mathrm{S}$ is a nonnegative real number. As usual, $H_{0}^{\mathrm{S}}(\Omega)$ stands for the closure in $H^{\mathrm{S}}(\Omega)$ of the space of infinitely differentiable functions with a compact support in $\Omega$, and $H^{-\mathrm{S}}(\Omega)$ stands for its dual space. For simplicity, we denote by $(\cdot, \cdot)$ the scalar product on $L^{2}(\Omega)$ or on $L^{2}(\Omega)^{\ell}$ for any positive integer $\ell$ and by $\|\cdot\|$ the associated norm. By extension, the duality pairing between any $H^{-\mathrm{S}}(\Omega)$ and $H_{0}^{\mathrm{S}}(\Omega), \mathrm{S}>0$, is also denoted by $(\cdot, \cdot)$. For any real number $\mathrm{S}$, we define $L^{2}\left(0, T ; H^{\mathrm{S}}(\Omega)\right)$, respectively $\mathscr{C}^{0}\left(0, T ; H^{\mathrm{S}}(\Omega)\right)$, as the space of square-integrable functions, respectively of continuous functions, with values in $H^{\mathrm{S}}(\Omega)$, and also $L^{2}\left(0, T ; H_{0}^{1}(\Omega)\right)$ as the space of square-integrable functions with values in $H_{0}^{1}(\Omega)$. Then, it is readily checked that equation (2.1) admits the equivalent variational formulation:

Find $u$ in $\mathscr{C}^{0}\left(0, T ; L^{2}(\Omega)\right) \cap L^{2}\left(0, T ; H_{0}^{1}(\Omega)\right)$ satisfying

$$
u_{\mid t=0}=u_{0} \quad \text { in } \Omega
$$

and such that, for a.e. $t$ in $] 0, T[$,

$$
\forall v \in H_{0}^{1}(\Omega), \quad\left(\partial_{t} u(t), v\right)+(\lambda(t) \nabla u(t), \nabla v)=(f(t), v) .
$$

It is well known ([LM, Ch. 3, §4], [Th, Ch. 4]) that, for any $f$ in $L^{2}\left(0, T ; H^{-1}(\Omega)\right)$ and $u_{0}$ in $L^{2}(\Omega)$, problem $(2.3)-(2.4)$ admits a unique solution. Moreover, let us introduce the norm

$$
[[v]](t)=\left(\|v(t)\|^{2}+\int_{0}^{t}\left\|\lambda^{\frac{1}{2}}(s) \nabla v(s)\right\|^{2} d s\right)^{\frac{1}{2}} .
$$

By taking $v$ equal to $u(t)$ in (2.4) and integrating on the interval ]0, $t$, we easily derive the following estimate, for all $t$ in $[0, T]$ :

$$
[[u]](t) \leq\left(\left\|u_{0}\right\|^{2}+\frac{1}{\lambda_{\min }}\|f\|_{L^{2}\left(0, t ; H^{-1}(\Omega)\right)}^{2}\right)^{\frac{1}{2}} .
$$


2.2. The time semi-discrete problem. In order to describe the (possibly adaptive) time discretization of equation (2.1), we introduce a partition of the interval $[0, T]$ into subintervals $\left[t_{n-1}, t_{n}\right], 1 \leq n \leq N$, such that $0=t_{0}<t_{1}<\cdots<t_{N}=T$. We denote by $\tau_{n}$ the length $t_{n}-t_{n-1}$, by $\tau$ the $N$-tuple $\left(\tau_{1}, \ldots, \tau_{N}\right)$ and by $|\tau|$ the maximum of the $\tau_{n}, 1 \leq n \leq N$. We also define the regularity parameter

$$
\sigma_{\tau}=\max _{2 \leq n \leq N} \frac{\tau_{n}}{\tau_{n-1}}
$$

With each family $\left(v^{n}\right)_{0 \leq n \leq N}$, we agree to associate the function $v_{\tau}$ on $[0, T]$ which is affine on each interval $\left[t_{n-1}, t_{n}\right], 1 \leq n \leq N$, and equal to $v^{n}$ at $t_{n}, 0 \leq n \leq N$. Equivalently, this function can be written, for $1 \leq n \leq N$, as

$$
\forall t \in\left[t_{n-1}, t_{n}\right], \quad v_{\tau}(t)=v^{n}-\frac{t_{n}-t}{\tau_{n}}\left(v^{n}-v^{n-1}\right) .
$$

For simplicity, we introduce the notation $\lambda^{n}=\lambda\left(t_{n}\right)$ and $f^{n}=f\left(t_{n}\right)$, which obviously requires the continuity of $f$ with respect to $t$. The semi-discrete problem issued from Euler's implicit scheme is now written as

$$
\begin{cases}\frac{u^{n}-u^{n-1}}{\tau_{n}}-\operatorname{div}\left(\lambda^{n} \nabla u^{n}\right)=f^{n} & \text { in } \Omega, 1 \leq n \leq N, \\ u^{n}=0 & \text { on } \partial \Omega, 1 \leq n \leq N, \\ u^{0}=u_{0} & \text { in } \Omega .\end{cases}
$$

Equivalently, it admits the variational formulation:

Find $\left(u^{n}\right)_{0 \leq n \leq N}$ in $L^{2}(\Omega) \times H_{0}^{1}(\Omega)^{N}$ satisfying

$$
u^{0}=u_{0} \quad \text { in } \Omega
$$

and such that, for $1 \leq n \leq N$,

$$
\forall v \in H_{0}^{1}(\Omega), \quad\left(u^{n}, v\right)+\tau_{n}\left(\lambda^{n} \nabla u^{n}, \nabla v\right)=\left(u^{n-1}, v\right)+\tau_{n}\left(f^{n}, v\right) .
$$

The existence and uniqueness of a solution $\left(u^{n}\right)_{0 \leq n \leq N}$ for any data $f$ in $\mathscr{C}^{0}\left(0, T ; H^{-1}(\Omega)\right)$ and $u_{0}$ in $L^{2}(\Omega)$ is now a simple consequence of the Lax-Milgram lemma.

Moreover, by using the notation $\lambda_{\min }^{n}=\inf _{x \in \bar{\Omega}} \lambda\left(\boldsymbol{x}, t_{n}\right)$ and taking $v$ equal to $u^{n}$ in (2.11), we easily derive the estimate of the quantity

$$
\left\|u^{n}\right\|^{2}+\tau_{n}\left\|\left(\lambda^{n}\right)^{\frac{1}{2}} \nabla u^{n}\right\|^{2} \leq\left\|u^{n-1}\right\|^{2}+\frac{\tau_{n}}{\lambda_{\min }^{n}}\left\|f^{n}\right\|_{H^{-1}(\Omega)}^{2} .
$$

We now define the norm on whole sequences $\left(v^{m}\right)_{1 \leq m \leq n}$ by

$$
\left[\left[\left(v^{m}\right)\right]\right]_{n}=\left(\left\|v^{n}\right\|^{2}+\sum_{m=1}^{n} \tau_{m}\left\|\left(\lambda^{m}\right)^{\frac{1}{2}} \nabla v^{m}\right\|^{2}\right)^{\frac{1}{2}} .
$$

Indeed, by summing up estimate (2.12) on $n$, we derive the semi-discrete analogue of $(2.6)$ :

$$
\left[\left[\left(u^{m}\right)\right]\right]_{n} \leq\left(\left\|u_{0}\right\|^{2}+\sum_{m=1}^{n} \frac{\tau_{m}}{\lambda_{\min }^{m}}\left\|f^{m}\right\|_{H^{-1}(\Omega)}^{2}\right)^{\frac{1}{2}} .
$$

The norm $\left[\left[\left(u^{m}\right)\right]\right]_{n}$ involved in this estimate is not equal to the norm $\left[\left[u_{\tau}\right]\right]\left(t_{n}\right)$ (see $(2.8)$ for the definition of the function $u_{\tau}$ ). However, when $u_{0}$ is supposed to be in $H^{1}(\Omega)$, they are equivalent, as proven in the next lemma. 
Lemma 2.1. Assume that the function $\lambda$ is continuously differentiable in time, with maximum value of $\partial_{t} \lambda$ on $\bar{\Omega} \times[0, T]$ denoted by $\mu_{\max }$. There exists a positive real number $\alpha_{0}$, equal to $\lambda_{\min } / 2 \mu_{\max }$, such the following equivalence property holds for $|\tau| \leq \alpha_{0}$ and for any family $\left(v^{n}\right)_{0 \leq n \leq N}$ in $H^{1}(\Omega)^{N+1}$

$$
\frac{1}{8}\left[\left[\left(v^{m}\right)\right]\right]_{n}^{2} \leq\left[\left[v_{\tau}\right]\right]^{2}\left(t_{n}\right) \leq \frac{3}{4}\left(1+\frac{3 \sigma_{\tau}}{2}\right)\left[\left[\left(v^{m}\right)\right]\right]_{n}^{2}+\frac{3}{4} \tau_{1}\left\|\left(\lambda^{1}\right)^{\frac{1}{2}} \nabla v^{0}\right\|^{2} .
$$

Proof. Owing to the definitions (2.5) and (2.13) of the norms, we have to compare the quantities

$$
X_{m}=\int_{t_{m-1}}^{t_{m}}\left\|\lambda^{\frac{1}{2}}(s) \nabla v_{\tau}(s)\right\|^{2} d s \quad \text { and } \quad Y_{m}=\tau_{m}\left\|\left(\lambda^{m}\right)^{\frac{1}{2}} \nabla v^{m}\right\|^{2} .
$$

First, thanks to the definition of $\mu_{\max }$, we have the standard estimate

$$
\forall s \in\left[t_{m-1}, t_{m}\right], \quad\left\|\lambda(s)-\lambda^{m}\right\|_{L^{\infty}(\Omega)} \leq \tau_{m} \mu_{\max },
$$

so that, when $|\tau| \leq \alpha_{0}$,

$$
\forall s \in\left[t_{m-1}, t_{m}\right], \quad \frac{1}{2} \leq\left\|\frac{\lambda(s)}{\lambda^{m}}\right\|_{L^{\infty}(\Omega)} \leq \frac{3}{2} .
$$

It can also be noted that, thanks to the definition of $v_{\tau}$ and for a.e. $\boldsymbol{x}$ in $\Omega$,

$$
\int_{t_{m-1}}^{t_{m}}\left|\nabla v_{\tau}(\boldsymbol{x}, s)\right|^{2} d s=\frac{\tau_{m}}{3}\left(\left|\nabla v^{m}(\boldsymbol{x})\right|^{2}+\left|\nabla v^{m-1}(\boldsymbol{x})\right|^{2}+\nabla v^{m}(\boldsymbol{x}) \cdot \nabla v^{m-1}(\boldsymbol{x})\right) \text {. }
$$

(1) By combining (2.16) and (2.17), we obtain

$$
X_{m} \geq \frac{\tau_{m}}{6}\left(\left\|\left(\lambda^{m}\right)^{\frac{1}{2}} \nabla v^{m}\right\|^{2}+\left\|\left(\lambda^{m}\right)^{\frac{1}{2}} \nabla v^{m-1}\right\|^{2}+\left(\left(\lambda^{m}\right)^{\frac{1}{2}} \nabla v^{m},\left(\lambda^{m}\right)^{\frac{1}{2}} \nabla v^{m-1}\right)\right) .
$$

So using the inequality $a b \geq-\frac{1}{4} a^{2}-b^{2}$ yields

$$
X_{m} \geq \frac{\tau_{m}}{8}\left\|\left(\lambda^{m}\right)^{\frac{1}{2}} \nabla v^{m}\right\|^{2}=\frac{1}{8} Y_{m},
$$

whence the first inequality in (2.15).

(2) Similarly, by combining (2.16) and (2.17) and using the inequality $a b \leq \frac{1}{2} a^{2}+\frac{1}{2} b^{2}$, we have

$$
X_{m} \leq \frac{3 \tau_{m}}{4}\left(\left\|\left(\lambda^{m}\right)^{\frac{1}{2}} \nabla v^{m}\right\|^{2}+\left\|\left(\lambda^{m}\right)^{\frac{1}{2}} \nabla v^{m-1}\right\|^{2}\right) .
$$

When $m$ is equal to 1 , we keep this inequality without modification. When $m$ is $>1$, we use an analogue of (2.16) to obtain

$$
X_{m} \leq \frac{3 \tau_{m}}{4}\left\|\left(\lambda^{m}\right)^{\frac{1}{2}} \nabla v^{m}\right\|^{2}+\frac{3 \tau_{m-1}}{4} \sigma_{\tau} \frac{3}{2}\left\|\left(\lambda^{m-1}\right)^{\frac{1}{2}} \nabla v^{m-1}\right\|^{2} .
$$

By summing up the previous lines on $m$, we derive the second inequality in (2.15).

In order to state the a priori error estimate, we observe that the family $\left(e^{n}\right)_{0 \leq n \leq N}$, with $e^{n}=u\left(t_{n}\right)-u^{n}$, satisfies $e^{0}=0$ and also, by integrating $\partial_{t} u$ between $t_{n-1}$ and $t_{n}$ and using equation (2.11) and equation (2.4) at time $t=t_{n}$,

$$
\forall v \in H_{0}^{1}(\Omega), \quad\left(e^{n}, v\right)+\tau_{n}\left(\lambda^{n} \nabla e^{n}, \nabla v\right)=\left(e^{n-1}, v\right)+\tau_{n}\left(\varepsilon^{n}, v\right),
$$

where the consistency error $\varepsilon^{n}$ is given by

$$
\left(\varepsilon^{n}, v\right)=\left(\frac{1}{\tau_{n}} \int_{t_{n-1}}^{t_{n}}\left(\partial_{t} u\right)(s) d s-\left(\partial_{t} u\right)\left(t_{n}\right), v\right) .
$$


So, applying (2.14) to this new problem, we derive the estimate: If the solution $u$ is such that $\partial_{t}^{2} u$ belongs to $L^{2}\left(0, T ; H^{-1}(\Omega)\right)$ and for $1 \leq n \leq N$,

$$
\left[\left[\left(u\left(t_{m}\right)-u^{m}\right)\right]\right]_{n} \leq \frac{2}{3 \lambda_{\min }^{\frac{1}{2}}}\left(\max _{1 \leq m \leq n} \tau_{m}\right)\left\|\partial_{t}^{2} u\right\|_{L^{2}\left(0, t_{n} ; H^{-1}(\Omega)\right)} .
$$

Thanks to Lemma 2.1, this also induces a similar bound for the norm $\left[\left[u-u_{\tau}\right]\right]\left(t_{n}\right)$.

2.3. The time and space discrete problem. We now describe the space discretization of problem $(2.10)-(2.11)$. For each $n, 0 \leq n \leq N$, let $\left(\mathcal{T}_{n h}\right)_{h}$ be a regular family of triangulations of $\Omega$ by closed triangles (in dimension $d=2$ ) or tetrahedra (in dimension $d=3$ ), in the usual sense that

- for each $h, \bar{\Omega}$ is the union of all elements of $\mathcal{T}_{n h}$,

- for each $h$, the intersection of two different elements of $\mathcal{T}_{n h}$, if not empty, is a corner, a whole edge or a whole face of both of them,

- the maximal ratio of the diameter of an element $K$ in $\mathcal{T}_{n h}$ to the diameter of its inscribed circle or sphere is bounded by a constant independent of $n$ and $h$.

As usual the discretization parameter $h$ denotes the maximal diameter of the elements of $\mathcal{T}_{n h}, 1 \leq n \leq N$, and, in all that follows, $c$ stands for a constant that may vary from a line to the next one but is always independent of $h$.

Let $k$ be a fixed positive integer. For each $h$, the discrete spaces are defined by

$$
X_{n h}=\left\{v_{h} \in H^{1}(\Omega) ; \forall K \in \mathcal{T}_{n h}, v_{h \mid K} \in \mathcal{P}_{k}(K)\right\}, \quad X_{n h}^{0}=X_{n h} \cap H_{0}^{1}(\Omega),
$$

where $\mathcal{P}_{k}(K)$ denotes the space of restrictions to $K$ of polynomials with $d$ variables and total degree $\leq k$. For each $t_{n}$, we also introduce an approximation $\lambda_{h}^{n}$ of $\lambda\left(t_{n}\right)$ which is polynomial on each $K$ in $\mathcal{T}_{n h}$ of degree smaller than a fixed integer $\ell$ and we assume that, for a fixed constant $c(\lambda)$ depending only on $\lambda$,

$$
\left\|\lambda\left(t_{n}\right)-\lambda_{h}^{n}\right\|_{L^{\infty}(\Omega)} \leq c(\lambda) h^{\ell+1}, \quad 1 \leq n \leq N .
$$

The fully discrete problem now reads

$$
\text { Find }\left(u_{h}^{n}\right)_{0 \leq n \leq N} \text { in } X_{0 h} \times \prod_{n=1}^{N} X_{n h}^{0} \text { satisfying }
$$

$$
u_{h}^{0}=\Pi_{h} u_{0} \quad \text { in } \Omega
$$

and such that, for $1 \leq n \leq N$,

$$
\forall v_{h} \in X_{n h}^{0}, \quad\left(u_{h}^{n}, v_{h}\right)+\tau_{n}\left(\lambda_{h}^{n} \nabla u_{h}^{n}, \nabla v_{h}\right)=\left(u_{h}^{n-1}, v_{h}\right)+\tau_{n}\left(f^{n}, v_{h}\right),
$$

where $\Pi_{h}$ denotes an appropriate interpolation or projection operator with values in $X_{0 h}$. For all $h$ such that $c(\lambda) h^{\ell+1}<\lambda_{\min }$ (or also for all $h$ when $\ell$ is equal to 0 or 1 and $\Pi_{h}$ is the Lagrange interpolation operator), the function $\lambda_{h}^{n}$ is positive on $\bar{\Omega}$. So, there also, the existence and uniqueness of a solution $\left(u_{h}^{n}\right)_{0 \leq n \leq N}$ for any data $f$ in $\mathscr{C}^{0}\left(0, T ; H^{-1}(\Omega)\right)$ and $u_{0}$ smooth enough for $\Pi_{h} u_{0}$ to be defined follow from the Lax-Milgram lemma. Moreover exactly the same arguments as for (2.14) lead to the estimate

$$
\left(\left\|u_{h}^{n}\right\|^{2}+\sum_{m=1}^{n} \tau_{m}\left\|\left(\lambda_{h}^{m}\right)^{\frac{1}{2}} \nabla u_{h}^{m}\right\|^{2}\right)^{\frac{1}{2}} \leq\left(\left\|\Pi_{h} u_{0}\right\|^{2}+\sum_{m=1}^{n} \frac{\tau_{m}}{\lambda_{\min }^{m}}\left\|f^{m}\right\|_{H^{-1}(\Omega)}^{2}\right)^{\frac{1}{2}} .
$$

Thus, if the parameter $h$ of the triangulations $\mathcal{T}_{n h}$ is such that

$$
c(\lambda) h^{\ell+1} \leq \frac{\lambda_{\min }^{n}}{2}, \quad 1 \leq n \leq N,
$$


it follows from (2.20) that

$$
\left[\left[\left(u_{h}^{m}\right)\right]\right]_{n} \leq \sqrt{2}\left(\left\|\Pi_{h} u_{0}\right\|^{2}+\sum_{m=1}^{n} \frac{\tau_{m}}{\lambda_{\min }^{m}}\left\|f^{m}\right\|_{H^{-1}(\Omega)}^{2}\right)^{\frac{1}{2}} .
$$

Remark. In practice, the triangulations $\mathcal{T}_{n h}$ for different values of $n$ are not completely independent. Indeed, since our aim is mesh adaptivity, each triangulation $\mathcal{T}_{n h}$ is derived from $\mathcal{T}_{n-1, h}$ by cutting some elements of $\mathcal{T}_{n-1, h}$ into a few number of subelements or gluing together some elements of $\mathcal{T}_{n-1, h}$ into a new triangle or tetrahedron. On the other hand, several triangulations can be employed at the same time $t_{n}$ for mesh adaptivity and, for simplicity, we use the notation $\mathcal{T}_{n h}$ only for the last one. This means that the first triangulation used at time $t_{n}$ is in fact the final one at time $t_{n-1}$, so that the term $u_{h}^{n-1}$ in (2.22) does not have to be re-interpolated here. Even if a large part of the interpolation nodes remains unchanged between two successive triangulations, we must take into account the re-interpolation error in the following analysis.

To conclude, let us assume that the operator $\Pi_{h}$ satisfies, for any $v$ in $H_{0}^{1}(\Omega) \cap$ $H^{\mathrm{S}+1}(\Omega), \mathrm{S}_{0} \leq \mathrm{S} \leq k$,

$$
\left\|v-\Pi_{h} v\right\|_{L^{2}(\Omega)}+h\left|v-\Pi_{h} v\right|_{H^{1}(\Omega)} \leq c h^{\mathrm{S}+1}\|v\|_{H^{\mathrm{S}+1}(\Omega)} .
$$

This is satisfied for instance when $\Pi_{h}$ is the standard Lagrange interpolation operator and $\mathrm{S}_{0}>\frac{d}{2}-1$. Then, assuming that the solution $\left(u^{n}\right)_{0 \leq n \leq N}$ of problem (2.9) belongs to $H^{\mathrm{S}+1}(\Omega)^{N+1}, \mathrm{~S}_{0} \leq \mathrm{S} \leq k$, and also that (2.23) holds, we have the following estimate (see [Th, Ch. 4] for more details on the proof):

$$
\begin{aligned}
{\left[\left[\left(u^{m}-u_{h}^{m}\right)\right]\right]_{n} \leq c\left(h ^ { \mathrm { S } } \left(h^{2}\right.\right.} & \left.+\lambda_{\max }|\tau|\right)^{\frac{1}{2}}\left(\sum_{m=0}^{n}\left\|u^{n}\right\|_{H^{\mathrm{S}+1}(\Omega)}^{2}\right)^{\frac{1}{2}} \\
& \left.+\frac{c(\lambda)}{\lambda_{\min }} h^{\ell+1}\left(\left\|u_{0}\right\|^{2}+\sum_{m=1}^{n} \frac{\tau_{m}}{\lambda_{\min }^{m}}\left\|f^{m}\right\|_{H^{-1}(\Omega)}^{2}\right)^{\frac{1}{2}}\right) .
\end{aligned}
$$

More sophiscated arguments allow for deriving an a priori estimate of the full error $\left[\left[\left(u\left(t_{m}\right)-u_{h}^{m}\right)\right]\right]_{n}$ (or equivalently of $\left[\left[u-u_{h \tau}\right]\right]\left(t_{n}\right)$; see Lemma 2.1) as a function of the regularity of $u$ only.

Usually $\ell$ is taken $\geq k-1$. Then, by combining estimates (2.18) and (2.26), we derive that the convergence order is 1 with respect to the time discretization and $k$ with respect to the space discretization.

\section{ERror INDICATORS FOR THE LINEAR HEAT EQUATION}

We are now interested in exhibiting error indicators and studying their equivalence with the error. We first describe the two types of indicators. Next we prove an upper bound for the error as a function of the Hilbertian sum of the indicators and finally a local upper bound for each indicator.

3.1. The error indicators. As already hinted, we work with two families of indicators, the first ones being linked to time discretization and the second ones to space discretization. Moreover, since we do not intend to work with local time steps, the first ones are local in time but global in space while the second ones are local with respect to both the time and space variables. 
For each $n, 1 \leq n \leq N$, we define the time error indicator

$$
\eta_{n}=\left(\frac{\tau_{n}}{3}\right)^{\frac{1}{2}}\left\|\left(\lambda_{h}^{n}\right)^{\frac{1}{2}} \nabla\left(u_{h}^{n}-u_{h}^{n-1}\right)\right\| .
$$

We refer to [JNT], [NSV] and [Pi] for analogous time error indicators, however not necessarily leading to the same estimates.

Next, for each element $K$ of $\mathcal{T}_{n h}$, we denote by $h_{K}$ the diameter of $K$ and by $\mathcal{E}_{K}$ the set of edges in dimension $d=2$, faces in dimension $d=3$, of $K$ that are not contained in $\partial \Omega$. Also let $h_{e}$ stand for the diameter of each $e$ in $\mathcal{E}_{K}$. As is standard, we introduce an approximation $f_{h}^{n}$ of $f^{n}$ which is polynomial of degree $\leq \ell$ on all elements of $\mathcal{T}_{n h}$. For each $n, 1 \leq n \leq N$, and any $K$ in $\mathcal{T}_{n h}$, we define the space error indicator

$$
\eta_{n, K}=h_{K}\left\|f_{h}^{n}-\frac{u_{h}^{n}-u_{h}^{n-1}}{\tau_{n}}+\operatorname{div}\left(\lambda_{h}^{n} \nabla u_{h}^{n}\right)\right\|_{L^{2}(K)}+\frac{1}{2} \sum_{e \in \mathcal{E}_{K}} h_{e}^{\frac{1}{2}}\left\|\left[\lambda_{h}^{n} \partial_{\nu} u_{h}^{n}\right]\right\|_{L^{2}(e)},
$$

where $\left[\lambda_{h}^{n} \partial_{\nu} u_{h}^{n}\right]$ denotes the jump of the normal derivative of $u_{h}^{n}$ times the quantity $\lambda_{h}^{n}$ through $e$. Note that these last error indicators are exactly those defined in $\mathrm{Pi}$ formula (2.11)] and in [BBHM formula (8)] as an extension of [BM] formula (24)] and also of their analogues for elliptic problems Ve1, §1.2].

Note that, in definition (3.2), $u_{h}^{n-1}$ could be replaced by $\Pi_{n h} u_{h}^{n-1}$, where $\Pi_{n h}$ denotes the orthogonal projection operator from $L^{2}(\Omega)$ on $X_{n h}^{0}$. We would rather avoid this, for the following two reasons:

- The term $u_{h}^{n-1}-\Pi_{n h} u_{h}^{n-1}$ is nonzero only in the triangles of $\mathcal{T}_{n h}$ which are the union of several elements of $\mathcal{T}_{n-1, h}$, i.e., in the places where the mesh is coarsened between $t_{n-1}$ and $t_{n}$, and the quantity $u_{h}^{n-1}$ can very easily be evaluated on the new mesh.

- The statement of at least one a posteriori estimate will be more complex in this case.

Since the functions $f_{h}^{n}$ and $\lambda_{h}^{n}$ are piecewise polynomial, these indicators can be computed explicitly. Moreover, since as already explained the triangulation $\mathcal{T}_{n h}$ is derived from $\mathcal{T}_{n-1, h}$ by locally refining or coarsening the mesh, evaluating the norms of the function $u_{h}^{n-1}$ on the new triangles or tetrahedra of $\mathcal{T}_{n h}$ is not very difficult. So computing the error indicators is not too expensive in comparison to solving problem (2.22).

3.2. An upper bound for the error. From now on, we assume that the function $\lambda$ satisfies (2.16) and (2.20) and is continuously differentiable in time. As previously, we denote by $\mu_{\max }$ the maximum value of $\partial_{t} \lambda$ on $\bar{\Omega} \times[0, T]$.

We now intend to bound the norms $\left[\left[u-u_{h \tau}\right]\right]\left(t_{n}\right), 1 \leq n \leq N$, as a function of the error indicators and some further terms involving the data (where of course $u_{h \tau}$ denotes the piecewise affine function equal to $u_{h}^{n}$ in each $t_{n}$ ). Here, we use the triangle inequality

$$
\left[\left[u-u_{h \tau}\right]\right]\left(t_{n}\right) \leq\left[\left[u-u_{\tau}\right]\right]\left(t_{n}\right)+\left[\left[u_{\tau}-u_{h \tau}\right]\right]\left(t_{n}\right),
$$

and we begin by evaluating $\left[\left[u-u_{\tau}\right]\right]\left(t_{n}\right)$. The proof of the estimate is rather technical (similar results are established in [NSV] in an abstract framework).

Let $\pi_{\tau}$ denote the interpolation operator with values in piecewise constant functions on $[0, T]$, defined as follows: for any function $v$ continuous on $[0, T], \pi_{\tau} v$ is 
constant on each interval $\left[t_{n-1}, t_{n}\right], 1 \leq n \leq N$, equal to $v\left(t_{n}\right)$. The following results are standard: if the function $v$ admits a derivative which belongs to $L^{2}(0, T)$, respectively which is continuous on $[0, T]$,

$$
\begin{aligned}
\left\|v-\pi_{\tau} v\right\|_{L^{2}\left(0, t_{n}\right)} \leq c\left(\max _{1 \leq m \leq n} \tau_{m}\right)\left\|v^{\prime}\right\|_{L^{2}\left(0, t_{n}\right)}, \\
\sup _{0 \leq s \leq t_{n}}\left|\left(v-\pi_{\tau} v\right)(s)\right| \leq c\left(\max _{1 \leq m \leq n} \tau_{m}\right) \sup _{0 \leq s \leq t_{n}}\left|v^{\prime}(s)\right| .
\end{aligned}
$$

Proposition 3.1. Assume the data $f$ continuous on $[0, T]$ with values in $H^{-1}(\Omega)$ and the function $u_{0}$ in $H^{1}(\Omega)$. Then, there exists a positive constant $\alpha_{1}$ such that, when both $|\tau|$ and $h$ are smaller than $\alpha_{1}$, the following a posteriori error estimate holds between the solution $u$ of problem (2.1) and the solution $\left(u^{n}\right)_{0 \leq n \leq N}$ of problem (2.9), for all $t_{n}, 1 \leq n \leq N$ :

$$
\begin{aligned}
{\left[\left[u-u_{\tau}\right]\right]\left(t_{n}\right) \leq } & c\left(\left[\left[u_{\tau}-u_{h \tau}\right]\right]\left(t_{n}\right)+\left(\sum_{m=1}^{n} \eta_{m}^{2}\right)^{\frac{1}{2}}\right. \\
& \left.+\left\|f-\pi_{\tau} f\right\|_{L^{2}\left(0, t_{n} ; H^{-1}(\Omega)\right)}+\left(1+\sigma_{\tau}\right)^{\frac{1}{2}} c_{n}\left(u_{0}, f\right)\left(\max _{1 \leq m \leq n} \tau_{m}\right)\right),
\end{aligned}
$$

where the constant $c_{n}\left(u_{0}, f\right)$ is given by

$$
c_{n}\left(u_{0}, f\right)=\left\|u_{0}\right\|+\tau_{1}^{\frac{1}{2}}\left\|\left(\lambda^{1}\right)^{\frac{1}{2}} \nabla u_{0}\right\|+t_{n}^{\frac{1}{2}}\|f\|_{\mathscr{C}^{0}\left(0, t_{n} ; H^{-1}(\Omega)\right)} .
$$

Proof. When "applying" equation (2.4) to the function $u_{\tau}$, we obtain for all $t$ in ]$\left.t_{n-1}, t_{n}\right]$ and $v$ in $H_{0}^{1}(\Omega)$

$$
\begin{aligned}
\left(\partial_{t} u_{\tau}(t), v\right)+\left(\lambda(t) \nabla u_{\tau}(t), \nabla v\right)= & \left(\frac{u^{n}-u^{n-1}}{\tau_{n}}, v\right)+\left(\left(\lambda(t)-\lambda^{n}\right) \nabla u_{\tau}(t), \nabla v\right) \\
& +\left(\lambda^{n}\left(\nabla u_{\tau}(t)-\nabla u^{n}\right), \nabla v\right)+\left(\lambda^{n} \nabla u^{n}, \nabla v\right),
\end{aligned}
$$

whence, from (2.11),

$$
\begin{aligned}
\left(\partial_{t} u_{\tau}(t), v\right)+ & \left(\lambda(t) \nabla u_{\tau}(t), \nabla v\right) \\
& =\left(f^{n}, v\right)+\left(\left(\lambda(t)-\lambda^{n}\right) \nabla u_{\tau}(t), \nabla v\right)+\left(\lambda^{n}\left(\nabla u_{\tau}(t)-\nabla u^{n}\right), \nabla v\right) .
\end{aligned}
$$

Thus, subtracting this line from equation (2.4) leads to

$$
\begin{aligned}
& \left(\partial_{t}\left(u-u_{\tau}\right)(t), v\right)+\left(\lambda(t) \nabla\left(u-u_{\tau}\right)(t), \nabla v\right) \\
& \quad=\left(f(t)-f^{n}, v\right)-\left(\left(\lambda(t)-\lambda^{n}\right) \nabla u_{\tau}(t), \nabla v\right)-\left(\lambda^{n}\left(\nabla u_{\tau}(t)-\nabla u^{n}\right), \nabla v\right) .
\end{aligned}
$$

We now take $v$ equal to $\left(u-u_{\tau}\right)(t)$, integrate this line on $\left[t_{n-1}, t_{n}\right]$ and sum up on the $n$. By noting that $u-u_{\tau}$ vanishes at $t=0$, this yields

$$
\begin{aligned}
\frac{1}{2}\left[\left[u-u_{\tau}\right]\right]^{2}\left(t_{n}\right) \leq \sum_{m=1}^{n}\left(\int_{t_{m-1}}^{t_{m}}\left(f(s)-f^{m}, v(s)\right) d s\right. & \\
& -\int_{t_{m-1}}^{t_{m}}\left(\left(\lambda(s)-\lambda^{m}\right) \nabla u_{\tau}(s), \nabla v(s)\right) d s \\
& \left.\quad-\int_{t_{m-1}}^{t_{m}}\left(\lambda^{m}\left(\nabla u_{\tau}(s)-\nabla u^{m}\right), \nabla v(s)\right) d s\right) .
\end{aligned}
$$

We now evaluate separately each term in the right-hand side of this equation. 
(1) It follows from the definition of $\pi_{\tau}$ that

$$
\begin{aligned}
& \left|\int_{t_{m-1}}^{t_{m}}\left(\left(f-f^{m}\right)(s), v(s)\right) d s\right| \\
& \quad \leq \frac{1}{\lambda_{\text {min }}^{\frac{1}{2}}}\left(\int_{t_{m-1}}^{t_{m}}\left\|\left(f-\pi_{\tau} f\right)(s)\right\|_{H^{-1}(\Omega)}^{2} d s\right)^{\frac{1}{2}}\left(\int_{t_{m-1}}^{t_{m}}\left\|\lambda^{\frac{1}{2}}(s) \nabla v(s)\right\|^{2} d s\right)^{\frac{1}{2}} .
\end{aligned}
$$

Moreover, note that

$$
\left(\sum_{m=1}^{n} \int_{t_{m-1}}^{t_{m}}\left\|\lambda^{\frac{1}{2}}(s) \nabla v(s)\right\|^{2} d s\right)^{\frac{1}{2}} \leq\left[\left[u-u_{\tau}\right]\right]\left(t_{n}\right) .
$$

(2) Concerning the second term, we have

$$
\begin{aligned}
\mid \int_{t_{m-1}}^{t_{m}}\left(\left(\lambda(s)-\lambda^{m}\right) \nabla\right. & \left.u_{\tau}(s), \nabla v(s)\right) d s \mid \\
\leq & \frac{1}{\lambda_{\min }} \sup _{t_{m-1} \leq s \leq t_{m}}\left\|\left(\lambda-\pi_{\tau} \lambda\right)(s)\right\|_{L^{\infty}(\Omega)} \\
& \times\left(\int_{t_{m-1}}^{t_{m}}\left\|\lambda^{\frac{1}{2}}(s) \nabla u_{\tau}(s)\right\|^{2} d s\right)^{\frac{1}{2}}\left(\int_{t_{m-1}}^{t_{m}}\left\|\lambda^{\frac{1}{2}}(s) \nabla v(s)\right\|^{2} d s\right)^{\frac{1}{2}},
\end{aligned}
$$

whence, from (3.4),

$$
\begin{aligned}
\mid \int_{t_{m-1}}^{t_{m}}((\lambda(s) & \left.\left.-\lambda^{m}\right) \nabla u_{\tau}(s), \nabla v(s)\right) d s \mid \\
& \leq \frac{\mu_{\max }}{\lambda_{\min }} \tau_{m}\left(\int_{t_{m-1}}^{t_{m}}\left\|\lambda^{\frac{1}{2}}(s) \nabla u_{\tau}(s)\right\|^{2} d s\right)^{\frac{1}{2}}\left(\int_{t_{m-1}}^{t_{m}}\left\|\lambda^{\frac{1}{2}}(s) \nabla v(s)\right\|^{2} d s\right)^{\frac{1}{2}} .
\end{aligned}
$$

The last term is bounded in (3.8), while it follows from (2.14) and (2.15) that, for $|\tau| \leq \alpha_{0}$

$$
\begin{aligned}
\left(\sum_{m=1}^{n} \int_{t_{m-1}}^{t_{m}}\left\|\lambda^{\frac{1}{2}}(s) \nabla u_{\tau}(s)\right\|^{2} d s\right)^{\frac{1}{2}} \leq\left[\left[u_{\tau}\right]\right]\left(t_{n}\right) & \\
& \leq c\left(1+\sigma_{\tau}\right)^{\frac{1}{2}}\left(\left\|u_{0}\right\|+\tau_{1}^{\frac{1}{2}}\left\|\left(\lambda^{1}\right)^{\frac{1}{2}} \nabla u_{0}\right\|+\left(\sum_{m=1}^{n} \tau_{m}\left\|f^{m}\right\|_{H^{-1}(\Omega)}^{2}\right)^{\frac{1}{2}}\right) .
\end{aligned}
$$

(3) Using a Cauchy-Schwarz inequality, we have

$$
\begin{aligned}
& \left|\int_{t_{m-1}}^{t_{m}}\left(\lambda^{m}\left(\nabla u_{\tau}(s)-\nabla u^{m}\right), \nabla v(s)\right) d s\right| \\
& \quad \leq\left(\int_{t_{m-1}}^{t_{m}}\left\|\left(\lambda^{m}\right)^{\frac{1}{2}} \nabla\left(u_{\tau}(s)-u^{m}\right)\right\|^{2} d s\right)^{\frac{1}{2}}\left(\int_{t_{m-1}}^{t_{m}}\left\|\left(\lambda^{m}\right)^{\frac{1}{2}} \nabla v(s)\right\|^{2} d s\right)^{\frac{1}{2}} .
\end{aligned}
$$

By adding and subtracting $\lambda(s)$, the last term can be bounded by

$$
\left(\int_{t_{m-1}}^{t_{m}}\left\|\left(\lambda^{m}\right)^{\frac{1}{2}} \nabla v(s)\right\|^{2} d s\right)^{\frac{1}{2}} \leq\left(1+\left(\frac{\mu_{\max }}{\lambda_{\min }}\right)^{\frac{1}{2}} \tau_{m}^{\frac{1}{2}}\right)\left(\int_{t_{m-1}}^{t_{m}} \|\left(\lambda^{\frac{1}{2}}(s) \nabla v(s) \|^{2} d s\right)^{\frac{1}{2}},\right.
$$

and moreover $\tau_{m} \leq \alpha_{1}$. By definition of $u_{\tau}$ (see (2.8)), we have

$$
\left(\int_{t_{m-1}}^{t_{m}}\left\|\left(\lambda^{m}\right)^{\frac{1}{2}} \nabla\left(u_{\tau}(s)-u^{m}\right)\right\|^{2} d s\right)^{\frac{1}{2}}=\left(\frac{\tau_{m}}{3}\right)^{\frac{1}{2}}\left\|\left(\lambda^{m}\right)^{\frac{1}{2}} \nabla\left(u^{m}-u^{m-1}\right)\right\| .
$$


Then, adding and subtracting $\nabla u_{h}^{m}$ and $\nabla u_{h}^{m-1}$ and using the previous arguments, we derive

$$
\begin{aligned}
\left(\int_{t_{m-1}}^{t_{m}} \|\left(\lambda^{m}\right)^{\frac{1}{2}}\right. & \left.\nabla\left(u_{\tau}(s)-u^{m}\right) \|^{2} d s\right)^{\frac{1}{2}} \\
\leq\left(\frac{\tau_{m}}{3}\right)^{\frac{1}{2}} & \left\|\left(\lambda^{m}\right)^{\frac{1}{2}} \nabla\left(u_{h}^{m}-u_{h}^{m-1}\right)\right\|+\left(\frac{\tau_{m}}{3}\right)^{\frac{1}{2}}\left\|\left(\lambda^{m}\right)^{\frac{1}{2}} \nabla\left(u^{m}-u_{h}^{m}\right)\right\| \\
& \quad+\left(\frac{\tau_{m}}{3}\right)^{\frac{1}{2}}\left(1+\frac{\mu_{\max }}{\lambda_{\min }} \tau_{m}\right)^{\frac{1}{2}}\left\|\left(\lambda^{m-1}\right)^{\frac{1}{2}} \nabla\left(u^{m-1}-u_{h}^{m-1}\right)\right\| .
\end{aligned}
$$

Using (2.15) yields that the sum over $m$ of the square of the last two terms can be bounded by $4\left[\left[u_{\tau}-u_{\tau h}\right]\right]^{2}\left(t_{n}\right)$. Finally, we observe that

$$
\left(\frac{\tau_{m}}{3}\right)^{\frac{1}{2}}\left\|\left(\lambda^{m}\right)^{\frac{1}{2}} \nabla\left(u_{h}^{m}-u_{h}^{m-1}\right)\right\| \leq \eta_{m}+\left(\frac{\tau_{m}}{3}\right)^{\frac{1}{2}}\left\|\left(\lambda^{m}-\lambda_{h}^{m}\right)^{\frac{1}{2}} \nabla\left(u_{h}^{m}-u_{h}^{m-1}\right)\right\|,
$$

and we derive from $(2.20)$ that, for $c(\lambda) h^{\ell+1} \leq \frac{1}{2} \lambda_{\min }$,

$$
\left\|\frac{\lambda^{m}-\lambda_{h}^{m}}{\lambda_{h}^{m}}\right\|_{L^{\infty}(\Omega)} \leq \frac{c(\lambda) h^{\ell+1}}{\lambda_{\min }-c(\lambda) h^{\ell+1}} \leq \frac{2 c(\lambda) h^{\ell+1}}{\lambda_{\min }} \leq 1,
$$

whence

$$
\left(\frac{\tau_{m}}{3}\right)^{\frac{1}{2}}\left\|\left(\lambda^{m}\right)^{\frac{1}{2}} \nabla\left(u_{h}^{m}-u_{h}^{m-1}\right)\right\| \leq 2 \eta_{m}
$$

Combining all this yields the desired result.

Using equations (2.4) and (2.11) yields that, for $1 \leq n \leq N$,

$$
\begin{aligned}
& \left\|\partial_{t}\left(u-u_{\tau}\right)\right\|_{L^{2}\left(t_{n-1}, t_{n} ; H^{-1}(\Omega)\right)} \\
& \quad \leq\left\|\lambda \nabla u-\lambda^{n} \nabla u^{n}\right\|_{L^{2}\left(t_{n-1}, t_{n} ; L^{2}(\Omega)\right)}+\left\|f-\pi_{\tau} f\right\|_{L^{2}\left(t_{n-1}, t_{n} ; H^{-1}(\Omega)\right)},
\end{aligned}
$$

whence

$$
\begin{aligned}
& \left\|\partial_{t}\left(u-u_{\tau}\right)\right\|_{L^{2}\left(t_{n-1}, t_{n} ; H^{-1}(\Omega)\right)} \\
& \quad \leq c\left(\left(\int_{t_{n_{1}}}^{t_{n}}\left\|\left(\lambda^{n}\right)^{\frac{1}{2}} \nabla\left(u_{\tau}(s)-u^{n}\right)\right\|^{2} d s\right)^{\frac{1}{2}}+\left\|\lambda^{\frac{1}{2}} \nabla\left(u-u_{\tau}\right)\right\|_{L^{2}\left(t_{n-1}, t_{n} ; L^{2}(\Omega)\right)}\right. \\
& \left.\quad+\left\|f-\pi_{\tau} f\right\|_{L^{2}\left(t_{n-1}, t_{n} ; H^{-1}(\Omega)\right)}+c_{n}\left(u_{0}, f\right)\left(\max _{1 \leq m \leq n} \tau_{m}\right)\right) .
\end{aligned}
$$

Since the first term in the right-hand side is evaluated in part (3) of the proof of Proposition 3.1, combining this estimate with Proposition 3.1 leads to the following corollary.

Corollary 3.2. If the assumptions of Proposition 3.1 are satisfied, there exists a positive constant $\alpha_{1}$ such that, when both $|\tau|$ and $h$ are smaller than $\alpha_{1}$, the following a posteriori error estimate holds between the solution $u$ of problem (2.1) and the solution $\left(u^{n}\right)_{0 \leq n \leq N}$ of problem $(2.9)$, for all $t_{n}, 1 \leq n \leq N$ :

$$
\begin{aligned}
& \left\|\partial_{t}\left(u-u_{\tau}\right)\right\|_{L^{2}\left(0, t_{n} ; H^{-1}(\Omega)\right)} \leq c\left(\left[\left[u_{\tau}-u_{h \tau}\right]\right]\left(t_{n}\right)+\left(\sum_{m=1}^{n} \eta_{m}^{2}\right)^{\frac{1}{2}}\right. \\
& \left.\quad+\left\|f-\pi_{\tau} f\right\|_{L^{2}\left(0, t_{n} ; H^{-1}(\Omega)\right)}+\left(1+\sigma_{\tau}\right)^{\frac{1}{2}} c_{n}\left(u_{0}, f\right)\left(\max _{1 \leq m \leq n} \tau_{m}\right)\right),
\end{aligned}
$$

where $c_{n}\left(u_{0}, f\right)$ is defined in (3.6). 
Remark. It can be observed from the previous proof that the error indicators $\eta_{n}$ are more naturally associated with the error between the solution of a space semidiscrete problem and the solution of problem $(2.21)-(2.22)$. However this semidiscrete problem is not easy to write when the triangulations $\mathcal{T}_{n h}$ depend on $n$, which is the case for mesh adaptivity.

The arguments for evaluating $\left[\left[u_{\tau}-u_{h \tau}\right]\right]\left(t_{n}\right)$ are simpler and rather standard, so we only give an abridged proof of the following result.

Proposition 3.3. Assume the data $f$ continuous on $[0, T]$ with values in $L^{2}(\Omega)$ and the function $u_{0}$ in $H^{1}(\Omega)$. Then, the following a posteriori error estimate holds between the solution $\left(u^{n}\right)_{0 \leq n \leq N}$ of problem $(2.10)-(2.11)$ and the solution $\left(u_{h}^{n}\right)_{0 \leq n \leq N}$ of problem $(2.21)-(2.22)$, for all $t_{n}, 1 \leq n \leq N$ :

$$
\begin{aligned}
{\left[\left[\left(u^{m}-u_{h}^{m}\right)\right]\right]_{n} \leq c\left(\sum_{m=1}^{n} \tau_{m} \sum_{K \in \mathcal{T}_{m h}}\right.} & \left.\left(\eta_{m, K}^{2}+h_{K}^{2}\left\|f^{m}-f_{h}^{m}\right\|_{L^{2}(K)}^{2}\right)\right)^{\frac{1}{2}} \\
& +c\left(\left\|u_{0}-\Pi_{h} u_{0}\right\|_{L^{2}(\Omega)}+c_{n}\left(u_{0}, f\right) h^{\ell+1}\right),
\end{aligned}
$$

where $c_{n}\left(u_{0}, f\right)$ is defined in (3.6).

Proof. For any $v$ in $H_{0}^{1}(\Omega)$ and any $v_{h}$ in $X_{n h}^{0}$, we have

$$
\begin{aligned}
& \left(u^{n}-u_{h}^{n}, v\right)+\tau_{n}\left(\lambda^{n} \nabla\left(u^{n}-u_{h}^{n}\right), \nabla v\right)=\left(u^{n-1}-u_{h}^{n-1}, v_{h}\right)+\left(u^{n}-u_{h}^{n}, v-v_{h}\right) \\
& \quad+\tau_{n}\left(\lambda^{n} \nabla u^{n}, \nabla\left(v-v_{h}\right)\right)-\tau_{n}\left(\lambda_{h}^{n} \nabla u_{h}^{n}, \nabla\left(v-v_{h}\right)\right)-\tau_{n}\left(\left(\lambda^{n}-\lambda_{h}^{n}\right) \nabla u_{h}^{n}, \nabla v\right) .
\end{aligned}
$$

By integrating by parts, we note that

$$
\begin{aligned}
\left(u^{n}-u_{h}^{n}, v-v_{h}\right)+\tau_{n}\left(\lambda^{n} \nabla u^{n}, \nabla\left(v-v_{h}\right)\right)-\tau_{n}\left(\lambda_{h}^{n} \nabla u_{h}^{n}, \nabla\left(v-v_{h}\right)\right) & \\
= & \left(u^{n-1}-u_{h}^{n-1}, v-v_{h}\right) \\
& +\tau_{n} \sum_{K \in \mathcal{T}_{n h}}\left(\int_{K}\left(f^{n}-\frac{u_{h}^{n}-u_{h}^{n-1}}{\tau_{n}}+\operatorname{div}\left(\lambda_{h}^{n} \nabla u_{h}^{n}\right)\right)(\boldsymbol{x})\left(v-v_{h}\right)(\boldsymbol{x}) d \boldsymbol{x}\right. \\
& \left.+\frac{1}{2} \sum_{e \in \mathcal{E}_{K}} \int_{e}\left[\lambda_{h}^{n} \partial_{\nu} u_{h}^{n}\right](r)\left(v-v_{h}\right)(r) d r\right) .
\end{aligned}
$$

We again use (2.20) to obtain

$$
\left|\tau_{n}\left(\left(\lambda^{n}-\lambda_{h}^{n}\right) \nabla u_{h}^{n}, \nabla v\right)\right| \leq \frac{c(\lambda)}{\lambda_{\min }^{\frac{1}{2}}} h^{\ell+1} \tau_{n}\left\|\left(\lambda^{n}\right)^{\frac{1}{2}} \nabla u_{h}^{n}\right\|\|\nabla v\| .
$$

By taking $v_{h}$ equal to the image of $v$ by a local regularization operator (see [Cl], [BG] or [Ve4] for the properties of such an operator) and using a triangle inequality, we derive

$$
\begin{aligned}
\left(u^{n}-u_{h}^{n}, v\right) & +\tau_{n}\left(\lambda^{n} \nabla\left(u^{n}-u_{h}^{n}\right), \nabla v\right) \leq\left(u^{n-1}-u_{h}^{n-1}, v\right) \\
& +c\left(\tau_{n} \sum_{K \in \mathcal{T}_{n h}}\left(\eta_{n, K}+h_{K}\left\|f^{n}-f_{h}^{n}\right\|_{L^{2}(K)}\right)\|v\|_{H^{1}\left(\Delta_{K}\right)}\right. \\
& \left.+h^{\ell+1} \tau_{n}\left\|\left(\lambda^{n}\right)^{\frac{1}{2}} \nabla u_{h}^{n}\right\|\|\nabla v\|\right),
\end{aligned}
$$


where $\Delta_{K}$ stand for the union of all elements in $\mathcal{T}_{n h}$ that share at least a corner with $K$. Finally we take $v$ equal to $u^{n}-u_{h}^{n}$, which gives

$$
\begin{aligned}
\left\|u^{n}-u_{h}^{n}\right\|^{2} & +\tau_{n}\left\|\left(\lambda^{n}\right)^{\frac{1}{2}} \nabla\left(u^{n}-u_{h}^{n}\right)\right\|^{2} \leq\left\|u^{n-1}-u_{h}^{n-1}\right\|^{2} \\
& +c\left(\tau_{n} \sum_{K \in \mathcal{T}_{n h}}\left(\eta_{n, K}^{2}+h_{K}^{2}\left\|f^{n}-f_{h}^{n}\right\|_{L^{2}(K)}^{2}\right)+h^{2(\ell+1)} \tau_{n}\left\|\left(\lambda^{n}\right)^{\frac{1}{2}} \nabla u_{h}^{n}\right\|^{2}\right) .
\end{aligned}
$$

By summing up this inequality on $n$ and using (2.24), we obtain the desired result.

There also the following corollary is derived in an easy way.

Corollary 3.4. If the assumptions of Proposition 3.3 are satisfied, the following a posteriori error estimate holds between the solution $\left(u^{n}\right)_{0 \leq n \leq N}$ of problem (2.10)(2.11) and the solution $\left(u_{h}^{n}\right)_{0 \leq n \leq N}$ of problem $(2.21)-(2.22)$, for all $t_{n}, 1 \leq n \leq N$ :

$$
\begin{aligned}
&\left(\sum_{m=1}^{n} \tau_{m}\left\|\frac{\left(u^{m}-u_{h}^{m}\right)-\left(u^{m-1}-u_{h}^{m-1}\right)}{\tau_{m}}\right\|_{H^{-1}(\Omega)}^{2}\right)^{\frac{1}{2}} \\
& \leq c\left(\sum_{m=1}^{n} \tau_{m} \sum_{K \in \mathcal{T}_{m h}}\left(\eta_{m, K}^{2}+h_{K}^{2}\left\|f^{m}-f_{h}^{m}\right\|_{L^{2}(K)}^{2}\right)\right)^{\frac{1}{2}} \\
& \quad+c\left(\left\|u_{0}-\Pi_{h} u_{0}\right\|_{L^{2}(\Omega)}+c_{n}\left(u_{0}, f\right) h^{\ell+1}\right),
\end{aligned}
$$

where $c_{n}\left(u_{0}, f\right)$ is defined in (3.6).

Combining the results of Propositions 3.1 and 3.3 with Lemma 2.1 leads to the full a posteriori error estimate.

Theorem 3.5. Assume the data $f$ continuous on $[0, T]$ with values in $L^{2}(\Omega)$ and the function $u_{0}$ in $H^{1}(\Omega)$. If moreover the regularity parameter $\sigma_{\tau}$ is bounded by a constant independent of $\tau$, there exists a positive constant $\alpha_{1}$ such that, when both $|\tau|$ and $h$ are smaller than $\alpha_{1}$, the following a posteriori error estimate holds between the solution $u$ of problem (2.1) and the solution $\left(u_{h}^{n}\right)_{0 \leq n \leq N}$ of problem $(2.21)-(2.22)$, for all $t_{n}, 1 \leq n \leq N$ :

$$
\begin{aligned}
& {\left[\left[u-u_{h \tau}\right]\right]\left(t_{n}\right) \leq c\left(\sum_{m=1}^{n}\left(\eta_{m}^{2}+\tau_{m} \sum_{K \in \mathcal{T}_{m h}}\left(\eta_{m, K}^{2}+h_{K}^{2}\left\|f^{m}-f_{h}^{m}\right\|_{L^{2}(K)}^{2}\right)\right)\right)^{\frac{1}{2}}} \\
& +c\left(\left\|u_{0}-\Pi_{h} u_{0}\right\|_{L^{2}(\Omega)}+\tau_{1}^{\frac{1}{2}}\left\|\nabla\left(u_{0}-\Pi_{h} u_{0}\right)\right\|\right. \\
& \left.\quad+\left\|f-\pi_{\tau} f\right\|_{L^{2}\left(0, t_{n} ; H^{-1}(\Omega)\right)}+c_{n}\left(u_{0}, f\right)\left(h^{\ell+1}+\max _{1 \leq m \leq n} \tau_{m}\right)\right),
\end{aligned}
$$

where $c_{n}\left(u_{0}, f\right)$ is defined in (3.6).

Estimate (3.15) seems fully optimal. Moreover, note that the limitation on $\tau$ and $h$ is due to the replacement in the discrete problems of $\lambda(t)$ by $\lambda^{n}$ and $\lambda_{h}^{n}$. When the function $\lambda$ is independent of $t$ and is polynomial of degree $\leq \ell$ on the whole domain $\Omega$, the following simpler estimate holds for all $\tau$ and $h$ whenever the 
assumptions of Theorem 3.5 hold:

$$
\begin{aligned}
{\left[\left[u-u_{h \tau}\right]\right]\left(t_{n}\right) \leq c\left(\sum _ { m = 1 } ^ { n } \left(\eta_{m}^{2}+\right.\right.} & \left.\left.\tau_{m} \sum_{K \in \mathcal{T}_{m h}}\left(\eta_{m, K}^{2}+h_{K}^{2}\left\|f^{m}-f_{h}^{m}\right\|_{L^{2}(K)}^{2}\right)\right)\right)^{\frac{1}{2}} \\
& +\left\|u_{0}-\Pi_{h} u_{0}\right\|_{L^{2}(\Omega)}+c\left\|f-\pi_{\tau} f\right\|_{L^{2}\left(0, t_{n} ; H^{-1}(\Omega)\right)} .
\end{aligned}
$$

The same estimate also holds for $\left\|\partial_{t}\left(u-u_{h \tau}\right)\right\|_{L^{2}\left(0, t_{n} ; H^{-1}(\Omega)\right)}$.

As usual in a posteriori estimates, the right-hand side of (3.15) contains the error indicators and several terms only involving the data. Even if these last terms are not explicitly taken into account in the adaptivity strategy that we propose, it is clear that the triangulation $\mathcal{T}_{0 h}$ (and also $\tau_{1}$ ) must be chosen fine enough in order that the approximation error concerning the term $u_{0}$ is smaller than a given tolerance. Analogous properties concerning the approximation of the data $f$ and $\lambda$ must be taken into account at each time step.

3.3. An upper bound for the error indicators. The idea is now to prove separate bounds for each indicator $\eta_{n}$ and $\eta_{n, K}$. We begin with the $\eta_{n}$.

Proposition 3.6. Assume the data $f$ continuous on $[0, T]$ with values in $H^{-1}(\Omega)$ and the function $u_{0}$ in $H_{0}^{1}(\Omega)$. The following estimate holds for the indicator $\eta_{n}$ defined in (3.1), $1 \leq n \leq N$ :

$$
\begin{aligned}
\eta_{n} \leq c_{n}^{\prime}(\lambda) & \left(\left[\left[u^{n}-u_{h}^{n}\right]\right]+\sigma_{\tau}^{\frac{1}{2}}\left[\left[u^{n-1}-u_{h}^{n-1}\right]\right]\right. \\
+c & \left(\left\|\lambda^{\frac{1}{2}} \nabla\left(u-u_{\tau}\right)\right\|_{L^{2}\left(t_{n-1}, t_{n} ; L^{2}(\Omega)\right)}+\left\|\partial_{t}\left(u-u_{\tau}\right)\right\|_{L^{2}\left(t_{n-1}, t_{n} ; H^{-1}(\Omega)\right)}\right. \\
& \left.\left.+\left\|f-\pi_{\tau} f\right\|_{L^{2}\left(t_{n-1}, t_{n} ; H^{-1}(\Omega)\right)}+c_{n}\left(u_{0}, f\right)\left(1+\sigma_{\tau}\right)^{\frac{1}{2}} \tau_{n}\right)\right)
\end{aligned}
$$

where $c_{n}\left(u_{0}, f\right)$ is defined in (3.6) and $c_{n}^{\prime}(\lambda)$ denotes the quantity

$$
c_{n}^{\prime}(\lambda)=\left(1+\frac{c(\lambda) h^{\ell+1}+\tau_{n} \mu_{\max }}{\lambda_{\min }}\right)^{\frac{1}{2}} .
$$

Proof. As previously, we use the triangle inequality

$$
\begin{aligned}
\eta_{n} & \leq\left(\frac{\tau_{n}}{3}\right)^{\frac{1}{2}}\left\|\left(\lambda_{h}^{n}\right)^{\frac{1}{2}} \nabla\left(u^{n}-u^{n-1}\right)\right\| \\
& +\left(\frac{\tau_{n}}{3}\right)^{\frac{1}{2}}\left\|\left(\lambda_{h}^{n}\right)^{\frac{1}{2}} \nabla\left(u^{n}-u_{h}^{n}\right)\right\|+\left(\frac{\tau_{n}}{3}\right)^{\frac{1}{2}}\left\|\left(\lambda_{h}^{n}\right)^{\frac{1}{2}} \nabla\left(u^{n-1}-u_{h}^{n-1}\right)\right\| .
\end{aligned}
$$

Thanks to (2.20), replacing $\lambda_{h}^{n}$ by $\lambda^{n}$ or $\lambda^{n-1}$ leads to

$$
\begin{aligned}
\eta_{n} \leq & \left(\frac{\tau_{n}}{3}\right)^{\frac{1}{2}}\left(1+\frac{c(\lambda) h^{\ell+1}}{\lambda_{\min }}\right)^{\frac{1}{2}}\left\|\left(\lambda^{n}\right)^{\frac{1}{2}} \nabla\left(u^{n}-u^{n-1}\right)\right\| \\
& +\left(\frac{\tau_{n}}{3}\right)^{\frac{1}{2}}\left(1+\frac{c(\lambda) h^{\ell+1}}{\lambda_{\min }}\right)^{\frac{1}{2}}\left\|\left(\lambda^{n}\right)^{\frac{1}{2}} \nabla\left(u^{n}-u_{h}^{n}\right)\right\| \\
& +\left(\frac{\tau_{n}}{3}\right)^{\frac{1}{2}}\left(1+\frac{c(\lambda) h^{\ell+1}+\tau_{n} \mu_{\max }}{\lambda_{\min }}\right)^{\frac{1}{2}}\left\|\left(\lambda^{n-1}\right)^{\frac{1}{2}} \nabla\left(u^{n-1}-u_{h}^{n-1}\right)\right\|,
\end{aligned}
$$

whence, using notation (3.18),

$\eta_{n} \leq\left(\frac{\tau_{n}}{3}\right)^{\frac{1}{2}} c_{n}^{\prime}(\lambda)\left\|\left(\lambda^{n}\right)^{\frac{1}{2}} \nabla\left(u^{n}-u^{n-1}\right)\right\|+c_{n}^{\prime}(\lambda)\left(\left[\left[u^{n}-u_{h}^{n}\right]\right]+\sigma_{\tau}^{\frac{1}{2}}\left[\left[u^{n-1}-u_{h}^{n-1}\right]\right]\right)$. 
Next, in order to evaluate the quantity $\left\|\left(\lambda^{n}\right)^{\frac{1}{2}} \nabla\left(u^{n}-u^{n-1}\right)\right\|$, we take $v$ in (3.7) equal to $u^{n}-u^{n-1}$ and integrate between $t_{n-1}$ and $t_{n}$. By noting that

$$
-\int_{t_{n-1}}^{t_{n}}\left(\lambda^{n}\left(\nabla u_{\tau}(s)-\nabla u^{n}\right), \nabla v\right) d s=\frac{\tau_{n}}{2}\left\|\left(\lambda^{n}\right)^{\frac{1}{2}} \nabla\left(u^{n}-u^{n-1}\right)\right\|^{2},
$$

we derive

$$
\begin{gathered}
\frac{\tau_{n}}{2}\left\|\left(\lambda^{n}\right)^{\frac{1}{2}} \nabla\left(u^{n}-u^{n-1}\right)\right\|^{2}=\int_{t_{n-1}}^{t_{n}}\left(\partial_{t}\left(u-u_{\tau}\right)(s), v\right) d s \\
+\int_{t_{n-1}}^{t_{n}}\left(\lambda(s) \nabla\left(u-u_{\tau}\right)(s), \nabla v\right) d s-\int_{t_{n-1}}^{t_{n}}\left(\left(f-\pi_{\tau} f\right)(s), v\right) d s \\
\quad+\int_{t_{n-1}}^{t_{n}}\left(\left(\lambda(s)-\lambda^{n}\right) \nabla u_{\tau}(s), \nabla v\right) d s .
\end{gathered}
$$

The first term is obviously bounded by (note that this requires that $u^{0}=u_{0}$ belongs to $\left.H_{0}^{1}(\Omega)\right)$

$$
\left|\int_{t_{n-1}}^{t_{n}}\left(\partial_{t}\left(u-u_{\tau}\right)(s), v\right) d s\right| \leq\left\|\partial_{t}\left(u-u_{\tau}\right)\right\|_{L^{2}\left(t_{n-1}, t_{n} ; H^{-1}(\Omega)\right)}\left(\frac{\tau_{n}}{\lambda_{\min }}\right)^{\frac{1}{2}}\left\|\left(\lambda^{n}\right)^{\frac{1}{2}} \nabla v\right\| .
$$

We also have

$$
\begin{aligned}
\mid \int_{t_{n-1}}^{t_{n}}(\lambda(s) & \left.\nabla\left(u-u_{\tau}\right)(s), \nabla v\right) d s \mid \\
\leq & \left(1+\frac{\tau_{n} \mu_{\max }}{\lambda_{\min }}\right)^{\frac{1}{2}} \tau_{n}^{\frac{1}{2}}\left\|\lambda^{\frac{1}{2}} \nabla\left(u-u_{\tau}\right)\right\|_{L^{2}\left(t_{n-1}, t_{n} ; L^{2}(\Omega)^{d}\right)}\left\|\left(\lambda^{n}\right)^{\frac{1}{2}} \nabla v\right\|,
\end{aligned}
$$

and similarly

$$
\left|\int_{t_{n-1}}^{t_{n}}\left(f-\pi_{\tau} f\right)(s) v d s\right| \leq\left(\frac{\tau_{n}}{\lambda_{\min }}\right)^{\frac{1}{2}}\left\|f-\pi_{\tau} f\right\|_{L^{2}\left(t_{n-1}, t_{n} ; H^{-1}(\Omega)\right)}\left\|\left(\lambda^{n}\right)^{\frac{1}{2}} \nabla v\right\| .
$$

The last term is bounded in the usual way:

$$
\begin{aligned}
& \left|\int_{t_{n-1}}^{t_{n}}\left(\left(\lambda(s)-\lambda^{n}\right) \nabla u_{\tau}(s), \nabla v\right) d s\right| \\
& \quad \leq \tau_{n}^{\frac{3}{2}} \frac{\mu_{\max }}{\lambda_{\min }}\left\|\lambda^{\frac{1}{2}} \nabla u_{\tau}\right\|_{L^{2}\left(t_{n-1}, t_{n} ; L^{2}(\Omega)^{d}\right)}\left\|\left(\lambda^{n}\right)^{\frac{1}{2}} \nabla v\right\|,
\end{aligned}
$$

and we use (2.14) and (2.15) to evaluate the norm of $u_{\tau}$. The desired estimate is thus obtained by inserting all these estimates into (3.19).

The most important property of estimate (3.17) is that, up to the constant $c_{n}\left(u_{0}, f\right)$, all terms in the right-hand side are local in time; more precisely, they involve the solution and the data only on the interval $\left[t_{n-1}, t_{n}\right]$. We now evaluate the indicators $\eta_{n, K}$. There, also, the arguments are very similar to those in $\left.\mathrm{BM}\right]$ or to their analogues for elliptic problems; however a further assumption is needed which is not too restrictive in the context of mesh adaptation. This is due to the fact that both $u_{h}^{n-1}$ and $u_{h}^{n}$ are involved in the definition of $\eta_{n, K}$ and are a priori not defined on the same mesh, and also that, as already hinted, we prefer not to replace $u_{h}^{n-1}$ by $\Pi_{n h} u_{h}^{n-1}$ in the definition of $\eta_{n, K}$. 
Proposition 3.7. Assume that, for $1 \leq n \leq N$, there exists a regular family of triangulations $\left(\mathcal{T}_{n h}^{*}\right)_{h}$ such that, for all $h$ and $n, 1 \leq n \leq N$, each element of $\mathcal{T}_{n-1, h}$ and $\mathcal{T}_{n h}$ is the union of at most $s$ elements of $\mathcal{T}_{n h}^{*}$ (where $s$ is bounded independently of $h$ and $n)$. Assume also the data $f$ continuous on $[0, T]$ with values in $L^{2}(\Omega)$ and the function $u_{0}$ in $H_{0}^{1}(\Omega)$. The following estimate holds for the indicator $\eta_{n, K}$ defined in (3.2), for all $K$ in $\mathcal{T}_{n h}, 1 \leq n \leq N$ :

$$
\begin{gathered}
\eta_{n, K} \leq c\left(\left\|\left(\lambda^{n}\right)^{\frac{1}{2}} \nabla\left(u^{n}-u_{h}^{n}\right)\right\|_{L^{2}\left(\omega_{K}\right)^{d}}+\left\|\frac{\left(u^{n}-u_{h}^{n}\right)-\left(u^{n-1}-u_{h}^{n-1}\right)}{\tau_{n}}\right\|_{H^{-1}\left(\omega_{K}\right)}\right. \\
\left.+h_{K}\left\|f^{n}-f_{h}^{n}\right\|_{L^{2}\left(\omega_{K}\right)}+c(\lambda) c_{n}\left(u_{0}, f\right) h^{\ell+1}\right),
\end{gathered}
$$

where $c_{n}\left(u_{0}, f\right)$ is defined in (3.6) and $\omega_{K}$ denotes the union of elements of $\mathcal{T}_{n h}$ that share at least an edge $(d=2)$ or a face $(d=3)$ with $K$.

Proof. We bound successively the two terms in $\eta_{n, K}$.

(1) We use (3.13) with $v_{h}$ equal to zero and $v$ equal to $v_{K}$, with

$v_{K}=\left(f_{h}^{n}-\frac{u_{h}^{n}-u_{h}^{n-1}}{\tau_{n}}+\operatorname{div}\left(\lambda_{h}^{n} \nabla u_{h}^{n}\right)\right) \psi_{K} \quad$ on $K \quad$ and $\quad v_{K}=0 \quad$ on $\Omega \backslash K$,

where $\psi_{K}$ denotes the bubble function on $K$. This leads to

$$
\begin{aligned}
\|\left(f_{h}^{n}-\right. & \left.\frac{u_{h}^{n}-u_{h}^{n-1}}{\tau_{n}}+\operatorname{div}\left(\lambda_{h}^{n} \nabla u_{h}^{n}\right)\right) \psi_{K}^{\frac{1}{2}} \|_{L^{2}(K)}^{2} \\
= & \int_{K}\left(\frac{\left(u^{n}-u_{h}^{n}\right)-\left(u^{n-1}-u_{h}^{n-1}\right)}{\tau_{n}}\right)(\boldsymbol{x}) v_{K}(\boldsymbol{x}) d \boldsymbol{x} \\
& +\int_{K} \lambda^{n}(\boldsymbol{x}) \nabla\left(u^{n}-u_{h}^{n}\right)(\boldsymbol{x}) \cdot \nabla v_{K}(\boldsymbol{x}) d \boldsymbol{x} \\
& -\int_{K}\left(f^{n}-f_{h}^{n}\right)(\boldsymbol{x}) v_{K}(\boldsymbol{x}) d \boldsymbol{x} \\
& +\int_{t_{n-1}}^{t_{n}} \int_{K}\left(\lambda^{n}-\lambda_{h}^{n}\right)(\boldsymbol{x}) \nabla u_{h}^{n}(\boldsymbol{x}) \cdot \nabla v_{K}(\boldsymbol{x}) d \boldsymbol{x} .
\end{aligned}
$$

Bounding the terms in the right-hand side follows the same arguments as in the previous proof. Moreover we note that $v_{K}$ is the product of $\psi_{K}$ by a polynomial of degree $\leq m=\max \{k, \ell, k+\ell-1\}$ on each element of $\mathcal{T}_{n h}^{*}$ and we use the inverse inequalities

$$
\forall w \in \mathcal{P}_{m}(K), \quad\|w\|_{L^{2}(K)} \leq c\left\|w \psi_{K}^{\frac{1}{2}}\right\|_{L^{2}(K)} \text { and }\|\nabla w\|_{L^{2}(K)^{d}} \leq c h_{K}^{-1}\|w\|_{L^{2}(K)} .
$$

This leads to the estimate for the first term.

(2) For any $e$ in $\mathcal{E}_{K}$, we denote by $K^{\prime}$ the other element of $\mathcal{T}_{n h}$ that contains $e$. We still take $v_{h}$ equal to zero in (3.13) but now $v$ is chosen equal to $v_{e}$, with

$$
v_{e}=R_{e, \kappa}\left(\left[\lambda_{h}^{n} \partial_{\nu} u_{h}^{n}\right] \psi_{e}\right) \quad \text { on } \kappa \in\left\{K, K^{\prime}\right\} \quad \text { and } \quad v_{e}=0 \quad \text { on } \Omega \backslash\left(K \cup K^{\prime}\right) \text {, }
$$

where $\psi_{e}$ is the bubble function on $e$ and $R_{e, \kappa}$ is a lifting operator of polynomials on $e$ vanishing on $\partial e$ into polynomials in $\kappa$ vanishing on $\partial \kappa \backslash e$ which is built by affine transformation from a similar operator on the reference element (see [BMV] $\S 8.2]$ for more details). Using the estimate established in the first part of the proof together with appropriate inverse inequalities leads to the desired result. 
Now, all the terms in (3.20) up to the last one are local in space and time. Moreover, when taking $v$ in (3.13) successively equal to $\sum_{K \in \mathcal{T}_{n h}} h_{K}^{2} v_{K}$ and to $\sum_{K \in \mathcal{T}_{n h}} \sum_{e \in \mathcal{E}_{K}} h_{e} v_{e}$ (with the notation of the previous proof), we obtain the global estimate

$$
\begin{aligned}
\left(\sum_{K \in \mathcal{T}_{n h}} \eta_{n, K}^{2}\right)^{\frac{1}{2}} \leq c & \left(\left\|\left(\lambda^{n}\right)^{\frac{1}{2}} \nabla\left(u^{n}-u_{h}^{n}\right)\right\|_{L^{2}(\Omega)^{d}}\right. \\
& +\left\|\frac{\left(u^{n}-u_{h}^{n}\right)-\left(u^{n-1}-u_{h}^{n-1}\right)}{\tau_{n}}\right\|_{H^{-1}(\Omega)} \\
& \left.+h\left\|f^{n}-f_{h}^{n}\right\|_{L^{2}(\Omega)}+c(\lambda) c_{n}\left(u_{0}, f\right) h^{\ell+1}\right) .
\end{aligned}
$$

When comparing this last estimate and Propositions 3.6 with Theorem 3.5 and Corollaries 3.2 and 3.4 , we observe that, up to the terms involving the data $u_{0}$ and $f$ and the function $\lambda$, the full sum of errors on $\Omega \times[0, T]$ is equivalent to the Hilbertian sum

$$
\left(\sum_{m=1}^{n}\left(\eta_{m}^{2}+\tau_{m} \sum_{K \in \mathcal{T}_{m h}} \eta_{m, K}^{2}\right)\right)^{\frac{1}{2}}
$$

even if the right-hand sides of (3.17) and (3.22) do not involve all the terms of the error. Moreover, all the terms of this sum can easily be computed once the discrete solution $\left(u_{h}^{n}\right)_{0 \leq n \leq N}$ is known.

\section{EXtension to THE SEMiLinear heAT EQUATION}

We first present the semilinear heat equation we are interested in and its discretization. Next we describe the error indicators linked to the full discrete problem and state estimates that allow us to compare them with the error.

4.1. The semilinear heat equation. In the same domain $\Omega$ as in Section 2 and for the same positive integer $T$, we now consider the equation

$$
\begin{cases}\partial_{t} u-\operatorname{div}(\alpha(u) \nabla u)=f & \text { in } \Omega \times] 0, T[, \\ u=0 & \text { on } \partial \Omega \times] 0, T[, \\ u_{\mid t=0}=u_{0} & \text { in } \Omega,\end{cases}
$$

where $\alpha$ is a given continuously differentiable function from $\mathbb{R}$ into $\mathbb{R}$ satisfying for some positive constants $\alpha_{\min }, \alpha_{\max }$ and $\beta_{\max }$,

$$
\forall \xi \in \mathbb{R}, \quad \alpha_{\min } \leq \alpha(\xi) \leq \alpha_{\max } \quad \text { and } \quad\left|\alpha^{\prime}(\xi)\right| \leq \beta_{\max }
$$

The data are the distribution $f$ and the function $u_{0}$, and the unknown is the function $u$.

The existence of a solution for any data $f$ in $L^{2}\left(0, T ; L^{2}(\Omega)\right)$ and $u_{0}$ in $L^{2}(\Omega)$ relies on a fixed point theorem and is proven for instance in [Li, Ch. VI, §3] (see also $\mathrm{AL}$ and $\mathrm{GM}$ ] for more complex problems). Moreover, when defining the norm

$$
[[v]](t)=\left(\|v(t)\|^{2}+\alpha_{\min } \int_{0}^{t}\|\nabla v(s)\|^{2} d s\right)^{\frac{1}{2}},
$$

it can be checked that, for all $t$ in $[0, T]$,

$$
[[u]](t) \leq\left(\left\|u_{0}\right\|^{2}+\frac{1}{\alpha_{\min }}\|f\|_{L^{2}\left(0, t ; H^{-1}(\Omega)\right)}^{2}\right)^{\frac{1}{2}} .
$$


4.2. The semi-discrete and discrete problems. We use here the same notation as in Section 2. We choose to work with a fully implicit discretization in time: the semi-discrete problem is written as

$$
\begin{cases}\frac{u^{n}-u^{n-1}}{\tau_{n}}-\operatorname{div}\left(\alpha\left(u^{n}\right) \nabla u^{n}\right)=f^{n} & \text { in } \Omega, 1 \leq n \leq N, \\ u^{n}=0 & \text { on } \partial \Omega, 1 \leq n \leq N, \\ u^{0}=u_{0} & \text { in } \Omega .\end{cases}
$$

The existence and uniqueness of a solution $\left(u^{n}\right)_{0 \leq n \leq N}$ for any data $f$ in $\mathscr{C}^{0}\left(0, T ; H^{-1}(\Omega)\right)$ and $u_{0}$ in $L^{2}(\Omega)$ can easily be derived from a fixed point theorem.

With the norm $\left[\left[\left(v^{m}\right)\right]\right]_{n}$ now being defined by

$$
\left[\left[\left(v^{m}\right)\right]\right]_{n}=\left(\left\|v^{n}\right\|^{2}+\alpha_{\min } \sum_{m=1}^{n} \tau_{m}\left\|\nabla v^{m}\right\|^{2}\right)^{\frac{1}{2}},
$$

we have the estimate

$$
\left[\left[\left(u^{m}\right)\right]\right]_{n} \leq\left(\left\|u_{0}\right\|^{2}+\frac{1}{\alpha_{\min }} \sum_{m=1}^{n} \tau_{m}\left\|f^{m}\right\|_{H^{-1}(\Omega)}^{2}\right)^{\frac{1}{2}} .
$$

Moreover, comparing the norms $\left[\left[u_{\tau}\right]\right]\left(t_{n}\right)$ and $\left[\left[\left(u^{m}\right)\right]\right]_{n}$ is easier than in the linear case, owing to the new definitions of these norms.

Lemma 4.1. The following equivalence property holds for any family $\left(v^{n}\right)_{0 \leq n \leq N}$ in $H^{1}(\Omega)^{N+1}$ :

$$
\frac{1}{4}\left[\left[\left(v^{m}\right)\right]\right]_{n}^{2} \leq\left[\left[v_{\tau}\right]\right]^{2}\left(t_{n}\right) \leq \frac{1}{2}\left(1+\sigma_{\tau}\right)\left[\left[\left(v^{m}\right)\right]\right]_{n}^{2}+\frac{\alpha_{\min }}{2} \tau_{1}\left\|\nabla v^{0}\right\|^{2} .
$$

We now introduce the Lagrange interpolation operator $\mathcal{I}_{h}$ with values in $X_{n h}$. We agree to denote by $\alpha_{h}$ the mapping defined on continuous functions $v$ on $\Omega$ by

$$
\alpha_{h}(v)=\min \left\{\max \left\{\mathcal{I}_{h}(\alpha(v)), \alpha_{\min }\right\}, \alpha_{\max }\right\} .
$$

Note that, when $k$ is equal to $1, \alpha_{h}(v)$ coincides with $\mathcal{I}_{h}(\alpha(v))$.

The fully discrete problem now reads

Find $\left(u_{h}^{n}\right)_{0 \leq n \leq N}$ in $X_{0 h} \times \prod_{n=1}^{N} X_{n h}^{0}$ satisfying

$$
u_{h}^{0}=\Pi_{h} u_{0} \quad \text { in } \Omega
$$

and such that, for $1 \leq n \leq N$ :

$$
\forall v_{h} \in X_{n h}^{0}, \quad\left(u_{h}^{n}, v_{h}\right)+\tau_{n}\left(\alpha_{h}\left(u_{h}^{n}\right) \nabla u_{h}^{n}, \nabla v_{h}\right)=\left(u_{h}^{n-1}, v_{h}\right)+\tau_{n}\left(f^{n}, v_{h}\right)
$$

For $1 \leq n \leq N$, the existence of a solution of each problem at time $t_{n}$ for $f^{n}$ in $H^{-1}(\Omega)$ and $u^{n-1}$ in $X_{n h}$ is now a simple consequence of Brouwer's fixed point theorem. Moreover, thanks to the choice of $\alpha_{h}$, the family $\left(u_{h}^{n}\right)_{0 \leq n \leq N}$ satisfies the following estimate, for $1 \leq n \leq N$,

$$
\left[\left[\left(u_{h}^{m}\right)\right]\right]_{n} \leq\left(\left\|\Pi_{h} u_{0}\right\|^{2}+\frac{1}{\alpha_{\min }} \sum_{m=1}^{n} \tau_{m}\left\|f^{m}\right\|_{H^{-1}(\Omega)}^{2}\right)^{\frac{1}{2}} .
$$

We refer to [Th, Ch. 13] for the a priori analysis of this discretization when the triangulations $\mathcal{T}_{n h}$ are independent of $n$. 
Remark. When solving (4.11), a quadrature formula is most often used to evaluate the nonbilinear term $\left(\alpha_{h}\left(u_{h}^{n}\right) \nabla u_{h}^{n}, \nabla v_{h}\right)$. The choice of the mapping $\alpha_{h}$ makes this calculus rather easy and, at least for low values of $k$, we work with a quadrature formula which is exact when applied to the product $\alpha_{h}\left(u_{h}^{n}\right) \nabla u_{h}^{n} \cdot \nabla v_{h}$, so that no error is induced by the use of this quadrature.

4.3. Error indicators, lower and upper bounds. The error indicators are now defined by analogy with the linear case, in the simplest possible way: for each $n$, $1 \leq n \leq N$,

$$
\eta_{n}=\left(\frac{\tau_{n}}{3}\right)^{\frac{1}{2}}\left\|\alpha_{h}\left(u_{h}^{n}\right)^{\frac{1}{2}} \nabla\left(u_{h}^{n}-u_{h}^{n-1}\right)\right\|,
$$

and for each $n, 1 \leq n \leq N$, and any $K$ in $\mathcal{T}_{n h}$,

$$
\begin{aligned}
\eta_{n, K}=h_{K} \| f_{h}^{n}-\frac{u_{h}^{n}-u_{h}^{n-1}}{\tau_{n}}+\operatorname{div}\left(\alpha_{h}^{n}\left(u_{h}^{n}\right)\right. & \left.\nabla u_{h}^{n}\right) \|_{L^{2}(K)} \\
& +\frac{1}{2} \sum_{e \in \mathcal{E}_{K}} h_{e}^{\frac{1}{2}}\left\|\alpha_{h}\left(u_{h}^{n}\right)\left[\partial_{\nu} u_{h}^{n}\right]\right\|_{L^{2}(e)} .
\end{aligned}
$$

The arguments for proving the two a posteriori error bounds for the error are similar to those used for the linear problem but involve a further use of Gronwall's lemma GR, Ch. V, Lemmas 1.8 and 2.4]. The first one requires some further regularity of the solution $\left(u^{n}\right)_{0 \leq n \leq N}$.

Proposition 4.2. Assume the data $f$ continuous on $[0, T]$ with values in $H^{-1}(\Omega)$ and the function $u_{0}$ in $H^{1}(\Omega)$. Assume also the solution $\left(u^{n}\right)_{0 \leq n \leq N}$ of problem (4.5) such that each $\nabla u^{n}$ belongs to $L^{p}(\Omega)^{d}$ for some $p>d$ and satisfies

$$
\sup _{0 \leq n \leq N}\left\|\nabla u^{n}\right\|_{L^{p}(\Omega)^{d}} \leq \rho^{*},
$$

for a constant $\rho^{*}$ depending only on the data $f$ and $u_{0}$ and the function $\alpha$. Then, there exists a constant $C^{*}$ only depending on $T, \alpha_{\min }, \alpha_{\max }, \beta_{\max }, p$ and $\rho^{*}$ such that the following a posteriori error estimate holds between the solution $u$ of problem (4.1) and the solution $\left(u^{n}\right)_{0 \leq n \leq N}$ of problem (4.5), for all $t_{n}, 1 \leq n \leq N$ :

$$
\left[\left[u-u_{\tau}\right]\right]\left(t_{n}\right) \leq C^{*}\left(\left[\left[u_{\tau}-u_{h \tau}\right]\right]\left(t_{n}\right)+\left(\sum_{m=1}^{n} \eta_{m}^{2}\right)^{\frac{1}{2}}+\left\|f-\pi_{\tau} f\right\|_{L^{2}\left(0, t_{n} ; H^{-1}(\Omega)\right)}\right),
$$

and that the following a posteriori error estimate holds between the solution $\left(u^{n}\right)_{0 \leq n \leq N}$ of problem (4.5) and the solution $\left(u_{h}^{n}\right)_{0 \leq n \leq N}$ of problem (4.10)- (4.11), for all $t_{n}, 1 \leq n \leq N$ :

$$
\begin{aligned}
{\left[\left[\left(u^{m}-u_{h}^{m}\right)\right]\right]_{n} \leq C^{*}\left(\sum _ { m = 1 } ^ { n } \tau _ { m } \sum _ { K \in \mathcal { T } _ { m h } } \left(\eta_{m, K}^{2}\right.\right.} & \left.\left.+h_{K}^{2}\left\|f^{m}-f_{h}^{m}\right\|_{L^{2}(K)}^{2}\right)\right)^{\frac{1}{2}} \\
& +c\left(\left\|u_{0}-\Pi_{h} u_{0}\right\|+c_{n}\left(u_{0}, f\right) h^{1-\frac{d}{p}}\right),
\end{aligned}
$$

where $c_{n}\left(u_{0}, f\right)$ is defined in (3.6).

Remark. The last term $c_{n}\left(u_{0}, f\right) h^{1-\frac{d}{p}}$ in (4.17) is a little disappointing when working with high values of $k$. Note however that it disappears when the function $\alpha$ is a truncated affine function (since $\alpha_{h}\left(u_{h}^{n}\right)$ thus coincides with $\alpha\left(u_{h}^{n}\right)$ ) and also 
when it is simple enough, for instance polynomial: in this last case, the term $\left(\alpha\left(u_{h}^{n}\right) \nabla u_{h}, \nabla v_{h}\right)$ can be computed explicitly, and using an approximation $\alpha_{h}$ is not necessary.

Remark. There also and with the same assumptions, estimates (4.16) and (4.17) still hold, respectively, for the quantities

$$
\left\|\partial_{t}\left(u-u_{\tau}\right)\right\|_{L^{2}\left(0, t_{n} ; H^{-1}(\Omega)\right)}
$$

and

$$
\left(\sum_{m=1}^{n} \tau_{m}\left\|\frac{\left(u^{m}-u_{h}^{m}\right)-\left(u^{m-1}-u_{h}^{m-1}\right)}{\tau_{m}}\right\|_{H^{-1}(\Omega)}^{2}\right)^{\frac{1}{2}} .
$$

Fortunately the upper bounds for the indicators are easier to establish.

Proposition 4.3. Assume the data $f$ continuous on $[0, T]$ with values in $W^{-1, p}(\Omega)$ for some $p>d$ and the function $u_{0}$ in $H_{0}^{1}(\Omega)$. Assume also the solution $\left(u^{n}\right)_{0 \leq n \leq N}$ of problem (4.7) such that each $\nabla u^{n}$ belongs to $L^{p}(\Omega)^{d}$ and satisfies (4.15). Then, there exists a constant $C_{0}$ only depending on $T, \alpha_{\min }, \alpha_{\max }, \beta_{\max }, p$ and $\rho^{*}$ such that the following estimates hold for the indicator $\eta_{n}$ defined in (4.13), $1 \leq n \leq N$ :

$$
\begin{aligned}
\eta_{n} \leq C_{0}\left(\left[\left[u^{n}-u_{h}^{n}\right]\right]+\sigma_{\tau}^{\frac{1}{2}}\left[\left[u^{n-1}-u_{h}^{n-1}\right]\right]\right. \\
+\left\|\nabla\left(u-u_{\tau}\right)\right\|_{L^{2}\left(t_{n-1}, t_{n} ; L^{2}(\Omega)^{d}\right)}+\left\|\partial_{t}\left(u-u_{\tau}\right)\right\|_{L^{2}\left(t_{n-1}, t_{n} ; H^{-1}(\Omega)\right)} \\
\left.\quad+\left\|f-\pi_{\tau} f\right\|_{L^{2}\left(t_{n-1}, t_{n} ; H^{-1}(\Omega)\right)}+c_{n}^{\prime}\left(f, \rho^{*}\right) \tau_{n}^{\min \left\{1,1+\frac{d}{4}-\frac{d}{p}\right\}}\right)
\end{aligned}
$$

where the constant $c_{n}^{\prime}\left(f, \rho^{*}\right)$ is given by

$$
c_{n}^{\prime}\left(f, \rho^{*}\right)=\max \left\{\alpha_{\max } \rho^{*}+\left\|f^{n}\right\|_{W^{-1, p}(\Omega)}, 2 \rho^{*}\right\},
$$

and, if the assumptions of Proposition 3.7 hold, for the indicator $\eta_{n, K}$ defined in (4.14), for all $K$ in $\mathcal{T}_{n h}, 1 \leq n \leq N$,

$$
\begin{array}{r}
\eta_{n, K} \leq C_{0}^{\prime}\left(\left\|\nabla\left(u^{n}-u_{h}^{n}\right)\right\|_{L^{2}\left(\omega_{K}\right)^{d}}+\left\|\frac{\left(u^{n}-u_{h}^{n}\right)-\left(u^{n-1}-u_{h}^{n-1}\right)}{\tau_{n}}\right\|_{H^{-1}\left(\omega_{K}\right)}\right. \\
\left.+h_{K}\left\|f^{n}-f_{h}^{n}\right\|_{L^{2}\left(\omega_{K}\right)}+c h^{1-\frac{d}{p}}\left\|\nabla u^{n}\right\|_{L^{2}\left(\omega_{K}\right)^{d}}\right)
\end{array}
$$

where $\omega_{K}$ denotes the union of elements of $\mathcal{T}_{n h}$ that share at least an edge $(d=2)$ or a face $(d=3)$ with $K$.

There also, all the estimates are optimal, and assumption (4.15) seems reasonable for smooth data (indeed, it can be checked that this property holds in the case of the linear equation (2.9) for any polygon or polyhedron $\Omega$ ). When summing up on $n$ the square of these estimates, we observe that, up to some terms concerning the data, the error is fully equivalent to the quantity

$$
\left(\sum_{m=1}^{n}\left(\eta_{m}^{2}+\tau_{m} \sum_{K \in \mathcal{T}_{m h}} \eta_{m, K}^{2}\right)\right)^{\frac{1}{2}}
$$

which is the same result as in the linear case. However the terms concerning the data can be larger. 


\section{REFERENCES}

[AL] H.W. Alt, S. Luckhaus, Quasilinear elliptic-parabolic differential equations, Math. Z. 183 (1983), 311-341. MR 85c:35059

[BBHM] A. Bergam, C. Bernardi, F. Hecht, Z. Mghazli, Error indicators for the mortar finite element discretization of a parabolic problem, Numerical Algorithms 34 (2003), 187201.

[BG] C. Bernardi, V. Girault, A local regularization operator for triangular and quadrilateral finite elements, SIAM J. Numer. Anal. 35 (1998), 1893-1916. MR 99g:65107

[BM] C. Bernardi, B. Métivet, Indicateurs d'erreur pour l'équation de la chaleur, Revue européenne des éléments finis 9 (2000), 425-438.

[BMV] C. Bernardi, B. Métivet, R. Verfürth, Analyse numérique d'indicateurs d'erreur, in Maillage et adaptation, P.-L. George ed., Hermès (2001), 251-278.

[BR] C. Bernardi, G. Raugel, Approximation numérique de certaines équations paraboliques non linéaires, R.A.I.R.O. Anal. Numér. 18 (1984), 237-285. MR 86a:65097

[BB1] M. Bieterman, I. Babuška, The finite element method for parabolic equations. I. A posteriori error estimation, Numer. Math. 40 (1982), 339-371. MR 85d:65052a

[BB2] M. Bieterman, I. Babuška, The finite element method for parabolic equations. II. A posteriori error estimation and adaptive approach, Numer. Math. 40 (1982), 373-406. MR 85d:65052b

[Cl] P. Clément, Approximation by finite element functions using local regularization, R.A.I.R.O. Anal. Numér. 9 (1975), 77-84. MR 53:4569

[EJ1] K. Eriksson, C. Johnson, Adaptive finite element methods for parabolic problems. I. A linear model problem, SIAM J. Numer. Anal. 28 (1991), 43-77. MR 91m:65274

[EJ2] K. Eriksson, C. Johnson, Adaptive finite element methods for parabolic problems. IV. Nonlinear problems, SIAM J. Numer. Anal. 32 (1995), 1729-1749. MR 96i:65081

[GM] M. Gabbouhy, Z. Mghazli, Un résultat d'existence de solution faible d'un système parabolique-elliptique non linéaire doublement dégénéré, Ann. Faculté des Sciences de Toulouse X (2001), 533-546. MR 2003g:35130

[GR] V. Girault, P.-A. Raviart, Finite Element Approximation of the Navier-Stokes Equations, Lecture Notes in Mathematics 749, Springer-Verlag (1979). MR 83b:65122

[JNT] C. Johnson, Y.-Y. Nie, V. Thomée, An a posteriori error estimate and adaptive timestep control for a backward Euler discretization of a parabolic problem, SIAM J. Numer. Anal. 27 (1990), 277-291. MR 91g:65199

[Li] J.-L. Lions, Équations différentielles opérationnelles et problèmes aux limites, Springer (1961). MR 27:3935

[LM] J.-L. Lions, E. Magenes, Problèmes aux limites non homogènes et applications, Vol. 1, Dunod (1968). MR 40:512

[NSV] R.H. Nochetto, G. Savaré, C. Verdi, A posteriori error estimates for variable time-step discretizations of nonlinear evolution equations, Comm. Pure Appl. Math. 53 (2000), 525-589. MR 2000k:65142

[Pi] M. Picasso, Adaptive finite elements for a linear parabolic problem, Comput. Methods Appl. Mech. Engrg. 167 (1998), 223-237. MR 2000b:65188

[PR] J. Pousin, J. Rappaz, Consistency, stability, a priori and a posteriori errors for PetrovGalerkin methods applied to nonlinear problems, Numer. Math. 69 (1994), 213-231. MR 95k:65111

[Th] V. Thomée, Galerkin Finite Element Methods for Parabolic Problems, Springer Series in Computational Mathematics 25, Springer (1997). MR 98m:65007

[Ve1] R. Verfürth, A Review of A Posteriori Error Estimation and Adaptive Mesh-Refinement Techniques, Wiley \& Teubner (1996).

[Ve2] R. Verfürth, A posteriori error estimates for nonlinear problems: $L^{r}\left(0, T ; W^{1, \rho}(\Omega)\right)-$ error estimates for finite element discretizations of parabolic equations, Numer. Meth. for PDE 14 (1998), 487-518. MR 99g:65099

[Ve3] R. Verfürth, A posteriori error estimates for nonlinear problems: $L^{r}\left(0, T ; L^{\rho}(\Omega)\right)$-error estimates for finite element discretizations of parabolic equations, Math. Comput. 67 (1998), 1335-1360. MR 99b:65120

[Ve4] R. Verfürth, Error estimates for some quasi-interpolation operators, Modél. Math. et Anal. Numér. 33 (1999), 695-713. MR 2001a:65149 
[Ve5] R. Verfürth, A posteriori error estimation techniques for non-linear elliptic and parabolic pdes, Revue européenne des éléments finis 9 (2000), 377-402.

Laboratoire Siano, Département de Mathématiques et d'Informatique, Faculté des Sciences, Université Ibn Tofail, B.P. 133, Kénitra, Maroc

Analyse Numérique, C.N.R.S. \& Université Pierre et Marie Curie,B.C. 187, 4 Place Jussieu, 75252 Paris Cedex 05, France

Laboratoire Siano, Département de Mathématiques et d'Informatique, Faculté des Sciences, Université Ibn Tofail, B.P. 133, Kénitra, Maroc 